\title{
SPITZER OBSERVATIONS OF THE YOUNG CORE-COLLAPSE SUPERNOVA REMNANT 1E0102-72.3: INFRARED EJECTA EMISSION AND DUST FORMATION
}

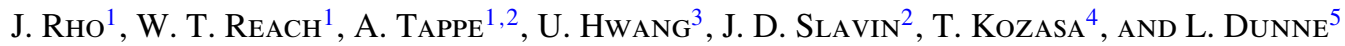 \\ ${ }^{1}$ Infrared Processing and Analysis Center, California Institute of Technology, Pasadena, CA 91125, USA; rho@ipac.caltech.edu \\ ${ }^{2}$ Harvard-Smithsonian Center for Astrophysics, MS 83, 60 Garden Street, Cambridge, MA 02138, USA \\ ${ }^{3}$ NASA Goddard Space Flight Center, Greenbelt, MD 20771, USA \\ ${ }^{4}$ Department of Cosmosciences, Graduate School of Science, Hokkaido University, Sapporo 060-0810, Japan \\ ${ }^{5}$ School of Physics and Astronomy, University of Nottingham, University Park, Nottingham NG7 2RD, UK \\ Received 2009 February 28; accepted 2009 April 27; published 2009 July 6
}

\begin{abstract}
We present Spitzer Infrared Spectrograph and Infrared Array Camera observations of the young supernova remnant E0102 (SNR 1E0102-7219) in the Small Magellanic Cloud. The infrared spectra show strong lines of Ne and $\mathrm{O}$, with the [Ne II] line at $12.8 \mu \mathrm{m}$ having a large velocity dispersion of $2000-4500 \mathrm{~km} \mathrm{~s}^{-1}$ indicative of fast-moving ejecta. Unlike the young Galactic SNR Cas A, E0102 lacks emission from Ar and Fe. Diagnostics of the observed [Ne III] line pairs imply that [Ne III] emitting ejecta have a low temperature of $650 \mathrm{~K}$, while [Ne V] line pairs imply that the infrared [Ne v] emitting ejecta have a high density of $\sim 10^{4} \mathrm{~cm}^{-3}$. We have calculated radiative shock models for various velocity ranges including the effects of photoionization. The shock model indicates that the [Ne v] lines come mainly from the cooling zone, which is hot and dense, whereas [Ne II] and [Ne III] come mainly from the photoionization zone, which has a low temperature of 400-1000 K. We estimate an infrared-emitting Ne ejecta mass of $0.04 M_{\odot}$ from the infrared observations, and discuss implications for the progenitor mass. The spectra also have a dust continuum feature peaking at $18 \mu \mathrm{m}$ that coincides spatially with the ejecta, providing evidence that dust formed in the expanding ejecta. The $18 \mu \mathrm{m}$ peak dust feature is fitted by a mixture of $\mathrm{MgSiO}_{3}$ and $\mathrm{Si}$ dust grains, while the rest of the continuum requires either carbon or $\mathrm{Al}_{2} \mathrm{O}_{3}$ grains. We measure the total dust mass formed within the ejecta of E0102 to be $\sim 0.014 M_{\odot}$. The dust mass in E0102 is thus a factor of a few smaller than that in Cas A. The composition of the dust is also different, showing relatively less silicate and likely no Fe-bearing dust, as is suggested by the absence of Fe-emitting ejecta.
\end{abstract}

Key words: dust, extinction - infrared: general - supernova remnants - supernovae: general

Online-only material: color figures

\section{INTRODUCTION}

Young supernova remnants (YSNRs) are the most viable astrophysical laboratories for the study of dust formation, nucleosynthesis of heavy elements, cosmic-ray acceleration, and shock physics. Of these, dust formation is the least well studied despite its invocation in efforts to explain some of the most important problems in astrophysics, including the isotopic anomalies of heavy elements in meteorites (Clayton 1982), dust formation in the early universe (Nozawa et al. 2003), the measured abundances of Galactic cosmic rays (Ellison et al. 1997), and the interstellar dust budget crisis (Jones et al. 1994).

Meteoritic and astronomical studies show that presolar, cosmic grains condense in the dense, warm stellar winds of evolved stars and in the ejecta of supernovae ( $\mathrm{SNe})$. Mantles of the preexisting dust in molecular clouds are vaporized as the forming stars and planetary systems heat them. A small fraction of the dust survives solar system formation without alteration, protected inside asteroids. The most abundant presolar grains are $\mathrm{SiC}$, nanodiamonds, amorphous silicates, fosterite and enstatite, and corundum $\left(\mathrm{Al}_{2} \mathrm{O}_{3}\right.$; Messenger et al. 2006). Some isotopic anomalies of heavy elements in meteorites have been attributed to the dust that had condensed deep within expanding $\mathrm{SNe}$ and some have the $r$-process composition commonly associated with Type II SNe (Clayton et al. 1997; Clayton \& Nittler 2004, and references therein).

Recent deep submillimeter observations have also shown there to be galaxies and QSOs with very large dust masses
( $>10^{8} M_{\odot}$ ) at $z>5$ (Wang et al. 2008; Beelen et al. 2006; Bertoldi et al. 2003). The timescales for low-mass (asymptotic giant branch (AGB)) stars to release their dust are too long to explain these high-redshift systems (Morgan \& Edmunds 2003). In contrast, SNe evolved from massive stars produce copious amounts of heavy elements and release them on short timescales. Theoretical modeling of the conditions in the SN ejecta indicates that Type II SNe are sources of dust formation and should produce substantial quantities of dust, on the order of a solar mass per explosion (Deneault et al. 2003, 2006; Todini $\&$ Ferrara 2001; Nozawa et al. 2003). Yet until very recently, there existed little observational evidence that this actually occurs.

There is now sufficient evidence for dust formation in corecollapse SNe. For SN 1987A, this includes dust emission, dust absorption, and a drop in line intensities for the refractory elements that signals that dust is being formed (Lucy et al. 1989, 1991). Formation of dust in the expanding ejecta of SN 1987A was explored theoretically (Kozasa et al. 1989, 1991). The Type II-P SN1999em also showed clear signs of dust formation (Elmhamdi et al. 2003). Detections of CO fundamentals with Spitzer observations of SN 2005af and Cas A further provide an indirect evidence of dust formation in SNe (Kotak et al. 2006; Rho et al. 2009).

For the young Galactic SNR, Cas A, Infrared Space Observatory (ISO) observations gave evidence for the association of the dust with the ejecta by requiring a mixture of dust grains that are not typical of the ISM, while showing strong spatial 

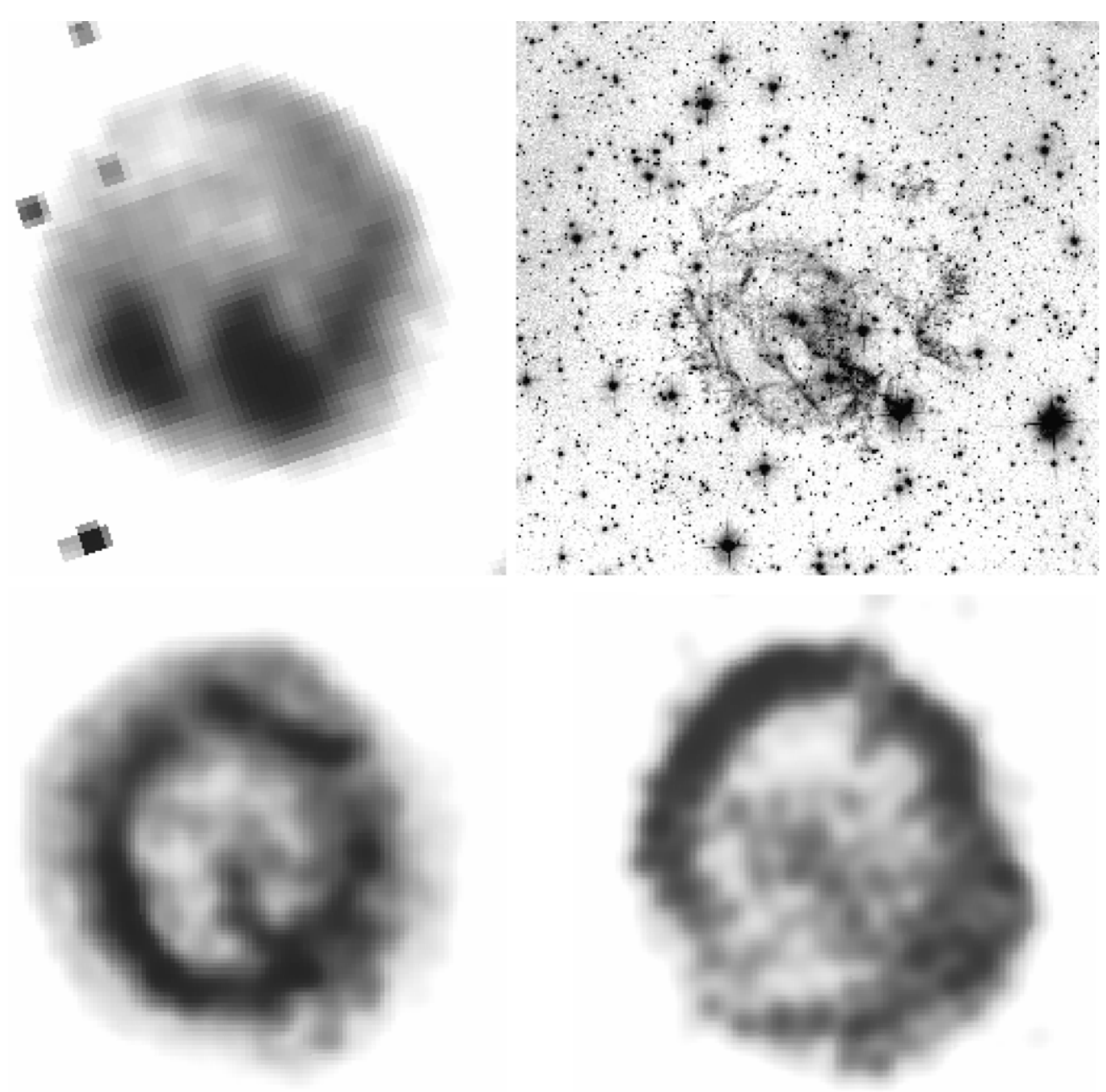

Figure 1. Four images of E0102 on the same angular scale (clockwise from upper left): infrared MIPS $24 \mu \mathrm{m}$ (Stanimirovic et al. 2005), HST (Finkelstein et al. 2006), ACTA radio at $6 \mathrm{~cm}$ (Amy \& Ball 1993), and Chandra X-rays (Flanagan et al. 2004). The brightest ring of radio emission traces the forward-shocked material, and the brightest ring of X-ray emission traces the reverse-shocked ejecta.

correlations between the high-velocity infrared emission lines, the dust continuum emission, and the optically emitting ejecta (Lagage et al. 1996; Arendt et al. 1999). Submillimeter observations of Cas A and Kepler with SCUBA (Dunne et al. 2003; Morgan et al. 2003) suggest the presence of large amounts of cold dust $\left(\sim 0.3-2 M_{\odot}\right.$ at $\left.15-20 \mathrm{~K}\right)$, but with some controversy related to the presence of foreground material. Krause et al. (2004) showed that much of the $160 \mu \mathrm{m}$ emission observed with Multiband Imaging Photometer for Spitzer (MIPS) is foreground material, suggesting there is no cold dust in Cas A. Wilson \& Batrla (2005), however, used CO emission toward the remnant to show that up to about a solar mass of dust could still be associated with the ejecta rather than with foreground material. New submillimeter polarimetry data confirms that a significant fraction $(>30 \%)$ of the submillimeter flux originates from within the remnant (Dunne et al. 2009). The case for dust formation in SN ejecta was strengthened by Spitzer observations of Cas A, for which line-free dust maps were found to remarkably resemble the infrared ejecta-line maps of [Ar II], [O VI], and [Ne II]; the estimated dust mass is between 0.02 and $0.054 M_{\odot}$ (Rho et al. 2008). A similar dust mass is reproduced by theoretical models of dust formation that include dust destruction and stochastic heating (Kozasa et al. 2009) and dust destruction (Bianchi \& Schneider 2007; Bianchi 2009) for Cas A.
Table 1

Summary of E0102 Properties ${ }^{\mathrm{a}}$

\begin{tabular}{lc}
\hline \hline Distance & $61.1 \mathrm{kpc}\left(1^{\prime} \sim 17.7801 \mathrm{pc}\right)$ \\
Angular size (diameter) & $44^{\prime \prime}$ \\
Physical size & $13.04 \mathrm{pc}$ \\
Shock radius & $6.52 \mathrm{pc}\left(22^{\prime \prime}\right)$ \\
Reverse shock radius & $4.45 \mathrm{pc}\left(15^{\prime \prime}\right)$ \\
Age & $1000 \mathrm{yr}, 2050 \pm 600 \mathrm{yr}$ \\
Extinction & $A_{\mathrm{v}}=0.08 \mathrm{mag}, N_{H}=2 \times 10^{20} \mathrm{~cm}^{-2}$ \\
\hline
\end{tabular}

Note. ${ }^{a}$ References are given in the text.

Another excellent source to study dust formation in $\mathrm{SNe}$ is the YSNR 1E 0102-7219 (SNR B0102-72.3; E0102 hereafter) in the Small Magellanic Cloud (SMC). As summarized in Table 1, it is located at R.A. $01^{\mathrm{h}} 04^{\mathrm{m}} 2^{\mathrm{s}} .57$ and decl. $-72^{\circ} 01^{\prime} 52^{\prime \prime} \cdot 3$ (J2000), and has a diameter of $44^{\prime \prime}$, which corresponds to $\sim 13.04 \mathrm{pc}$ for a distance of $61.1 \mathrm{kpc}$ to the SMC (Hilditch et al. 2005; Keller \& Wood 2006). Various wavelength images of E0102 are shown in Figure 1. Highly enriched abundances of oxygen and neon relative to carbon and magnesium (Blair et al. 2000), as is characteristic of massive progenitors, place E0102 in the class of "oxygen-rich" SNRs, along with Cas A. The optical ejecta in E0102 have substantial velocities that exceed $1000 \mathrm{~km} \mathrm{~s}^{-1}$ 
and imply a dynamic age of about $1000 \mathrm{yr}$ (Tuohy 1983). The $\mathrm{X}$-ray proper motion of the blast wave measured by Hughes et al. (2000) is also consistent with this age. More recent Hubble Space Telescope (HST) measurements of the ejecta filament proper motions imply a kinematic age of $2050 \pm 600 \mathrm{yr}$ that is a factor of 2 higher (Finkelstein et al. 2006), but the proper motion measurements are consistent within their quoted errors. The composition of the optically emitting ejecta suggests a massive progenitor that underwent either a Type Ib (Blair et al. 2000) or Type Ic (Flanagan et al. 2004) SN explosion. The optical expansion rates place E0102 currently in transition from free expansion to the Sedov phase. The low extinction toward E0102 compared with that of Galactic SNRs makes it possible to observe the infrared emission without contamination by emission from clouds along the line of sight (as seen, for example, in Cas A; Krause et al. 2004). The low metallicity environment of the SMC also makes E0102 a useful Type II prototype to compare with SNRs at higher redshifts.

In the infrared, the $24 \mu \mathrm{m}$ Spitzer MIPS image of E0102 presented by Stanimirovic et al. (2005) shows a filled morphology with two prominent elongated filaments that resemble structures seen in the X-ray image; the remnant was not detected at 8 or $70 \mu \mathrm{m}$. The infrared emission appears to be mainly associated with reverse shocks of the hot X-ray gas. An infrared emission peak around $24 \mu \mathrm{m}$ would suggest the presence of hot dust in E0102 with $T \sim 120 \mathrm{~K}$, with most of the dust being centrally located, based on the $24 \mu \mathrm{m}$ image. Assuming that the dust originates in the ISM and using typical ISM dust absorption coefficients (Draine \& Lee 1984), the estimated dust mass is $8 \times 10^{-4} M_{\odot}$. Even if all the dust were actually formed in the explosion, this dust mass is lower than might be expected based on some theoretical models. Most importantly, since the $24 \mu \mathrm{m}$ emission contains both line and continuum, not photometry but spectroscopy is required to estimate the mass and composition of dust as was demonstrated by Rho et al. (2008).

In this paper, we present Spitzer spectral and imaging observations of E0102 with Infrared Spectrograph (IRS) and Infrared Array Camera (IRAC). We find that the infrared ejecta emission is dominated by $\mathrm{Ne}$ and $\mathrm{O}$ and detect a dust feature peaking at $18 \mu \mathrm{m}$ which is spatially coincident with the ejecta emission. A high portion of the dust clearly comes from ejecta, rather than being all associated with circumstellar medium (CSM)/ interstellar medium (ISM) material. We show that some of dust is at lower temperature and a total dust mass is higher than inferred from earlier observations. We discuss physical conditions of infrared-emitting $\mathrm{Ne}$ and its inferred ejecta mass using emission line diagnostics and shock models.

\section{OBSERVATIONS}

We performed an IRS staring observation toward the southeastern shell of E0102 (R.A. $01^{\mathrm{h}} 04^{\mathrm{m}} 04.04$ and decl. $-72^{\circ} 02^{\prime} 00^{\prime \prime}$. 5, J2000, see Figure 2) as a part of our YSNR Spitzer GO program (PI: Rho). The long low (LL: 15-40 $\mu \mathrm{m}$ ) IRS data were taken on 2005 August 14 with six cycles of 30 s exposure time; this yields a total exposure time of $360 \mathrm{~s}$ for the first and second staring positions. The short low (SL: 5-15 $\mu \mathrm{m}$ ) IRS observations were made with three cycles of $60 \mathrm{~s}$ exposure time and one cycle covers two dither positions; this yields a total exposure time of $360 \mathrm{~s}$ per sky position.

The IRS spectra were processed using the S15.3 pipeline products as follows. First, we removed rogue pixels (bad pixels mainly due to cosmic rays) by subtracting nearby empty sky fields with the same exposure time. We took the empty sky

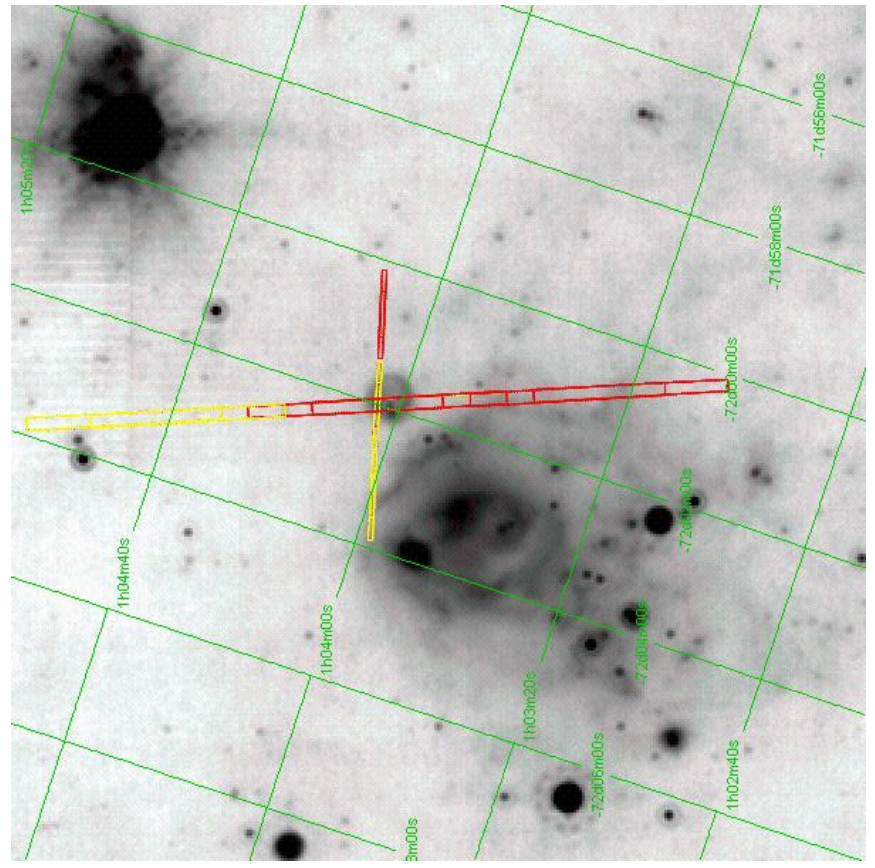

Figure 2. IRS slit coverage is superposed on an MIPS $24 \mu \mathrm{m}$ image (Stanimirovic et al. 2005) of E0102. North is to the right and east is up. The large diffuse source located southwest of E0102 is the [H II] regions of N76.

(A color version of this figure is available in the online journal.)

data from our observation in the alternate orders or from archival data (AORs 14706944, 14707712, 14708480, and 14708824). Any remaining rogue pixels were removed by using the synchrotron self-Compton (SSC) tool IRSCLEAN. ${ }^{6}$ Second, we extracted the spectrum using SPICE, and removed fringes. Third, we applied aperture and slit-loss corrections for diffuse emission (also see Tappe et al. 2006). The reduced spectrum is shown in Figure 3. Additionally, we subtracted a local background spectrum using the relatively emission-free regions right outside the SNR in our slit data (specifically, the region outside the eastern side of E0102 at R.A. 16.060, decl. -72.039). The background-subtracted spectrum is shown in Figure 4. Comparison of the two spectra is described in Section 3.1. The staring mode yields four coadded spectral images (positions 1 and 2, LL1 and LL2) which we merged to make a position-wavelength map as shown in Figure 5. The location of E0102 on this map is marked by the appearance of both continuum emission and strong [Ne III] $(15.55 \mu \mathrm{m})$ and weak [Ne v] $(23.3 \mu \mathrm{m})$ emission lines.

We also used archival IRS mapping data from the IRS Legacy SMC program (PI: Bolatto). These maps were made for large regions of the SMC and included E0102. The observations used one cycle of $30 \mathrm{~s}$ exposure time with 5 arcsec overlap, yielding a total of $60 \mathrm{~s}$ per sky position. These mapping data were six times shallower than our GO IRS staring-mode data.

We made a deep IRAC observation as a part of our GO program. The IRAC observations were made for a full array with $30 \mathrm{~s}$ frame time and a nine position random dither pattern, yielding $36 \mathrm{~s}$ exposure per sky position. The data were taken on 2005 June 12. IRAC bands cover the wavelength ranges 3.2$4.0 \mu \mathrm{m}, 4.0-5.0 \mu \mathrm{m}, 5.0-6.4 \mu \mathrm{m}$, and 6.4-9.4 $\mu \mathrm{m}$, respectively, with an FWHM of 1".7.

\footnotetext{
6 http://ssc.spitzer.caltech.edu/archanaly/contributed/
} 
E0102

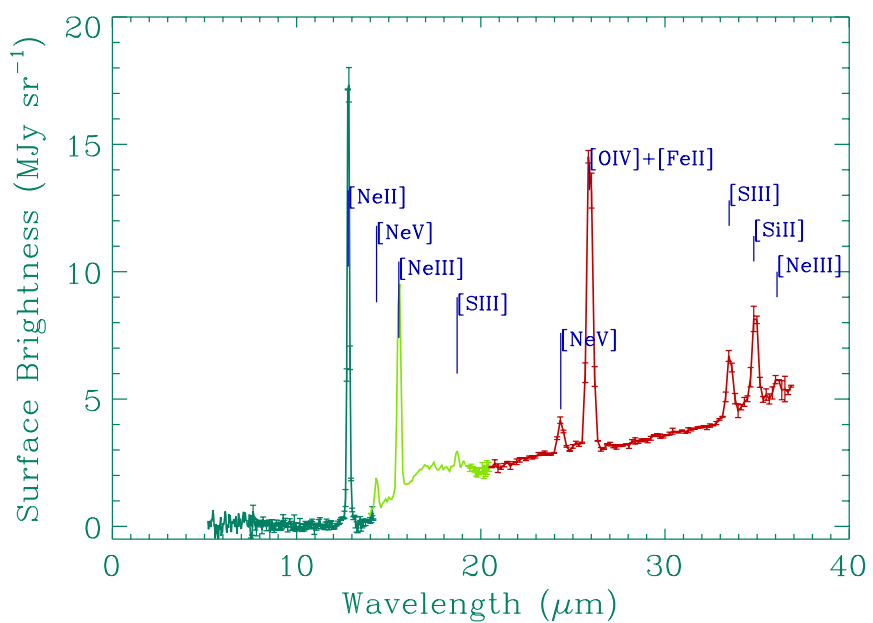

Figure 3. Spitzer IRS spectrum of E0102.

(A color version of this figure is available in the online journal.)

\section{RESULTS}

\subsection{IRS Spectra: Continuum and Ejecta Lines}

The IRS spectra of E0102 from the southeastern shell in Figure 3 show both line and continuum emission. The detected lines include [Ne II] $(12.8 \mu \mathrm{m}),[\mathrm{Ne} \mathrm{V}](14.3 \mu \mathrm{m}),[\mathrm{Ne}$ III] (15.5 $\mu \mathrm{m}),[\mathrm{Ne} \mathrm{v}](24.3 \mu \mathrm{m})$, [O IV] and/or [Fe II] $(26 \mu \mathrm{m})$, and [Ne III] $(36.07 \mu \mathrm{m})$. The local background subtraction effectively removed the [S III] $(33.5 \mu \mathrm{m})$, [Si II] $(34.8 \mu \mathrm{m})$, and [S III] $(18.7 \mu \mathrm{m})$ lines, but this background is likely to be contaminated by the nearby $\mathrm{H}$ II region of N76. When we generated a [Si II] map from the IRS cube, E0102 appeared as a [Si II] emitter. The lack of clean local background data thus makes it unclear whether there are actually any $\mathrm{S}$ and $\mathrm{Si}$ lines associated with E0102. The position-wavelength LL maps in Figure 5 show that the SNR E0102 emits continuum emission, [Ne v], [Ne III], and [O IV] (or [Fe II]). The $26 \mu \mathrm{m}$ blend is likely to be dominated by [O IV], and unlikely to contain any [Fe II], because other [Fe II] lines such as those at 17.9, 6.7, and $5.3 \mu \mathrm{m}$ are not detected. The infrared spectrum of E0102 is thus dominated by $\mathrm{Ne}$ and $\mathrm{O}$ lines.

The detected lines and their brightnesses are summarized in Table 2. We measured the line brightnesses using two methods: a Gaussian fitting, and integration of line fluxes over wavelength ranges. The latter is required in order to account for red and blueshifted velocity dispersions. To obtain unreddened line brightness, we used an extinction value $\left(A_{\mathrm{v}}\right)$ of $0.08 \mathrm{mag}$ (Blair et al. 1989), which is equivalent to $2(<8) \times 10^{20} \mathrm{~cm}^{-2}$. This value is consistent with those of X-ray measurements (Sasaki et al. 2006). The extinction corrected values of $A_{\lambda}$ are also shown in Table 1 . Because the extinction is very small, the unreddened line brightnesses differ by less than $0.3 \%$.

The E0102 spectrum shows a dust feature between 16 and $20 \mu \mathrm{m}$ peaking at $18.5 \mu \mathrm{m}$ (we call it the $18 \mu \mathrm{m}$ peak dust feature, hereafter). This feature occurs in the wavelength range of a prominent silicate feature, but it is very broad and extends to shorter wavelengths. We present a multicomposition fit in Section 4.1.

\subsection{IRAC Images}

Faint emission, particularly from the southeastern shell, is seen in our IRAC $8 \mu \mathrm{m}$ image of E0102; the $8 \mu \mathrm{m}$

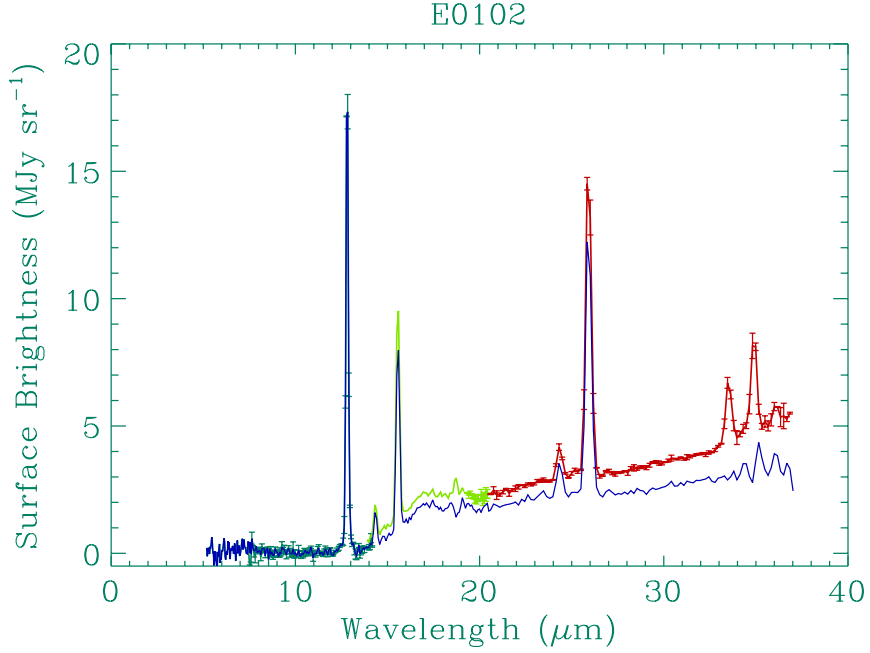

Figure 4. Spectrum before (top), and the spectrum (bottom) after local background subtraction.

(A color version of this figure is available in the online journal.)

emission corresponds well with the MIPS $24 \mu \mathrm{m}$ emission, as shown in Figure 6. The flux range in the image is $0.15-$ $0.2 \mathrm{MJy} \mathrm{sr}^{-1}$. However, there is also some $8 \mu \mathrm{m}$ polycyclic aromatic hydrocarbons (PAHs) emission from the nearby H II region of N76 to the southwestern side of the SNR and a large patch of diffuse emission outside the southwestern shell of the SNR. We did not detect $8 \mu \mathrm{m}$ emission with IRS, but IRS has poorer sensitivity than IRAC, with a limit of $0.24 \mathrm{MJy} \mathrm{sr}^{-1}$ for our IRS staring observation compared with $0.15 \mathrm{MJy} \mathrm{sr}^{-1}$ for IRAC. Because the IRAC flux is measured relative to the nearby background, we believe that the IRAC $8 \mu \mathrm{m}$ emission detected toward E0102 comes from the SNR. Moreover, dust emission is expected in the ejecta and any emission from E0102 at $8 \mu \mathrm{m}$ is likely to be continuum emission. A deep IRAC image, or a more sensitive observation with future missions may be helpful in unambiguously determining if E0102 does emit at $8 \mu \mathrm{m}$. No emission of E0102 at 3.6, 4.5, and $5.8 \mu \mathrm{m}$ is detected.

\subsection{Line and Dust Maps}

We generated line and continuum maps using the IRS mapping data, which covered the SNR E0102 and the nearby H II region N76. The maps are shown in Figure 7. The line maps were generated for [Ne II], [Ne III], [O IV], and [S III] by subtracting the continuum baselines at neighboring wavelengths. Infrared ejecta emission from [Ne II], [Ne III], [O IV], and [Si II] (Figure 7) coincides with the optical [O III] ejecta emission as well as with $\mathrm{X}$-ray ejecta emission. In spite of the lower angular resolution for the infrared images, this can be seen clearly in the overlay of the [Ne II] image with the optical [O III] contours (from Blair et al. 1989) and the X-ray Chandra image (Gaetz et al. 2000) in Figure 8. The infrared emission is strong at the southeastern shell and center, both of which coincide with ejecta emission seen in X-ray and optical images. The infrared line emission is weaker in the northern and northwestern regions, which are, however, also associated with X-ray-emitting ejecta. The ejectaline profile of the northwestern region shows significant line broadening (see Section 3.4 for details). The infrared continuum image is most similar to the infrared [O IV] $+[\mathrm{Fe}$ II] (at $26 \mu \mathrm{m}$ ) line image, except in the northwestern shell. However, this continuum emission at the northwestern shell nicely follows the X-ray ejecta shell as shown in Figure 7(d), and is located 


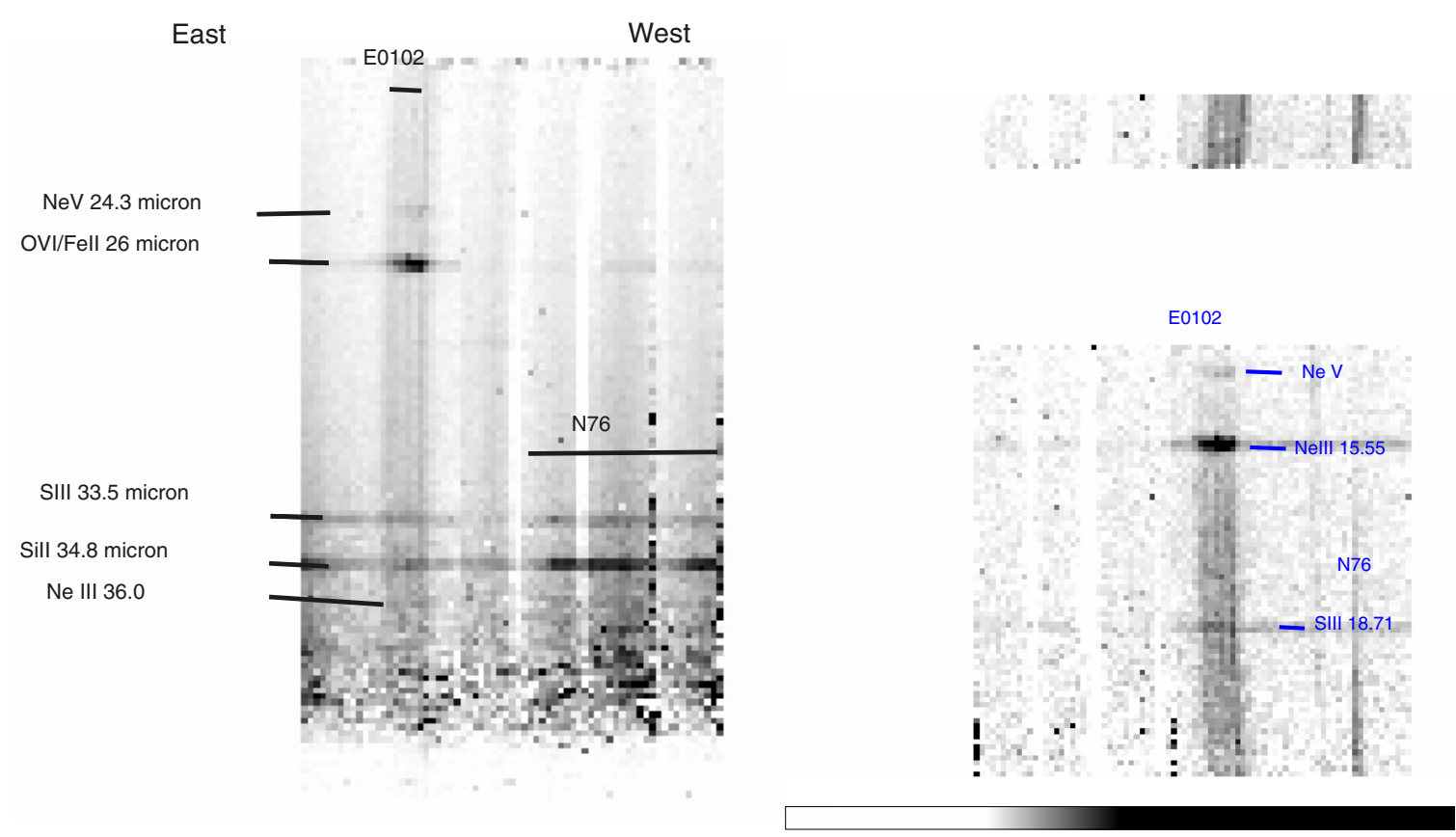

Figure 5. Merged LL position-wavelength images (left: LL2, right: LL1); $X$-axis is spatial direction along the slit covering E0102 and N76 and $y$-axis is wavelength. The image shows that E0102 emits continuum, [Ne v], [Ne II], and [O IV] lines.

(A color version of this figure is available in the online journal.)

Table 2

Observed Spectral Line Brightnesses

\begin{tabular}{|c|c|c|c|c|c|}
\hline $\begin{array}{l}\text { Wavelength } \\
(\mu \mathrm{m})\end{array}$ & Line & $\begin{array}{c}\text { FWHM } \\
(\mu \mathrm{m})\end{array}$ & $\begin{array}{l}\text { Surface Brightness }{ }^{\mathrm{a}} \\
\left(\mathrm{erg} \mathrm{s}^{-1} \mathrm{~cm}^{-2} \mathrm{sr}^{-1}\right)\end{array}$ & $\begin{array}{l}\text { Surface Brightness }{ }^{\mathrm{b}} \\
\left(\mathrm{erg} \mathrm{s}^{-1} \mathrm{~cm}^{-2} \mathrm{sr}^{-1}\right)\end{array}$ & $A_{\lambda}$ \\
\hline $12.8145 \pm 0.0010$ & {$[\mathrm{Ne} I I]$} & $0.1529 \pm 0.0023$ & $5.918 \mathrm{E}-05 \pm 8.774 \mathrm{E}-07$ & $5.525 \mathrm{E}-05$ & 0.0022 \\
\hline $14.3475 \pm 0.0023$ & {$[\mathrm{Ne} v]$} & $0.1797 \pm 0.0053$ & $3.974 \mathrm{E}-06 \pm 1.834 \mathrm{E}-07$ & $3.633 \mathrm{E}-06$ & 0.0016 \\
\hline $15.5665 \pm 0.0014$ & {$[\mathrm{Ne}$ III] } & $0.2027 \pm 0.0032$ & $2.354 \mathrm{E}-05 \pm 1.079 \mathrm{E}-06$ & $2.491 \mathrm{E}-05$ & 0.0019 \\
\hline $24.3410 \pm 0.0059$ & {$[\mathrm{Ne} v]$} & $0.3395 \pm 0.0142$ & $2.260 \mathrm{E}-06 \pm 9.676 \mathrm{E}-08$ & $2.364 \mathrm{E}-06$ & 0.0016 \\
\hline $25.9132 \pm 0.0006$ & {$[\mathrm{O}$ IV $]+[\mathrm{Fe}$ II $]$} & $0.3430 \pm 0.0015$ & $2.077 \mathrm{E}-05 \pm 9.442 \mathrm{E}-08$ & $2.116 \mathrm{E}-05$ & 0.0015 \\
\hline $33.5160 \pm 0.0010$ & {$[\mathrm{~S}$ III $]$} & $0.3489 \pm 0.0040$ & $2.394 \mathrm{E}-06 \pm 3.023 \mathrm{E}-08$ & $2.490 \mathrm{E}-06$ & 0.0011 \\
\hline $34.8901 \pm 0.0010$ & [Si II] & $0.3459 \pm 0.0027$ & $3.429 \mathrm{E}-06 \pm 2.920 \mathrm{E}-08$ & $3.612 \mathrm{E}-06$ & 0.0010 \\
\hline $36.0783 \pm 0.0041$ & {$[\mathrm{Ne} I I I]$} & $0.2565 \pm 0.0197$ & $4.329 \mathrm{E}-07 \pm 3.595 \mathrm{E}-08$ & $5.206 \mathrm{E}-07$ & 0.0009 \\
\hline
\end{tabular}

Notes.

a Estimated using a Gaussian fitting.

${ }^{\mathrm{b}}$ Estimated using integration of line fluxes over wavelength ranges.

inside the forward-shocked material traced by the radio shell (see Figure 1). The dust emission is thus seen to be spatially correlated with the ejecta emission.

We compared the line and continuum maps of E0102 with those of the H II region, N76, to its southeast (see Figure 9), in order to examine their infrared colors and to assess the effect of background contamination by N76. E0102 shows stronger [Ne II] and weaker [O IV] emission than N76. The Ne lines of the SNR are thus confidently measured. The continuum and [S III] emission of N76 is strongest at its outer edge, while [O IV] is strongest at the inner shell of N76, as shown in Figure 9. Since E0102 has its strongest [Ne II], [O IV], and dust continuum emission in the southern and eastern parts, we are confident that the $\mathrm{Ne}, \mathrm{O}$, and continuum measurements are not contaminated by N76. The [S III] line image shows that the SNR also emits [S III] emission as well as the H II region. Therefore, the [S III] line in the IRS spectra likely belongs to the SNR. We use the spectrum without local background subtraction in Figure 4 for the spectrum of E0102, but the difference from the local background-subtracted spectrum is used in estimating the uncertainty of the spectrum.

Infrared ejecta seem to most closely correlate with optical emission. Figure 8 demonstrates the correlation among the $\mathrm{X}$-ray, optical and three of our infrared ejecta maps. Infrared [O IV] is a fair direct comparison to the optical [O III] line maps, but the [O IV] image has a factor of few worse angular resolution than that of the [Ne II] image. [Ne II] and [O IV] correlate with optical [O III] images in the southwestern shell and center. It is likely the infrared ejecta are denser, cooler ejecta than the optical ejecta. In the future, the infrared and optical data should be combined to more accurately estimate of the ejecta mass, because a wide range of ionization states are observed in the remnant. However, it will be more meaningful to combine them within similar resolution images, since the ejecta knots have very small scales not only in optical but also in infrared as shown in the case of Cas A (Ennis et al. 2006). The dust continuum emission correlates with [O III] and X-ray emission for most of E0102 as well as with infrared ejecta in the southwestern and central parts 


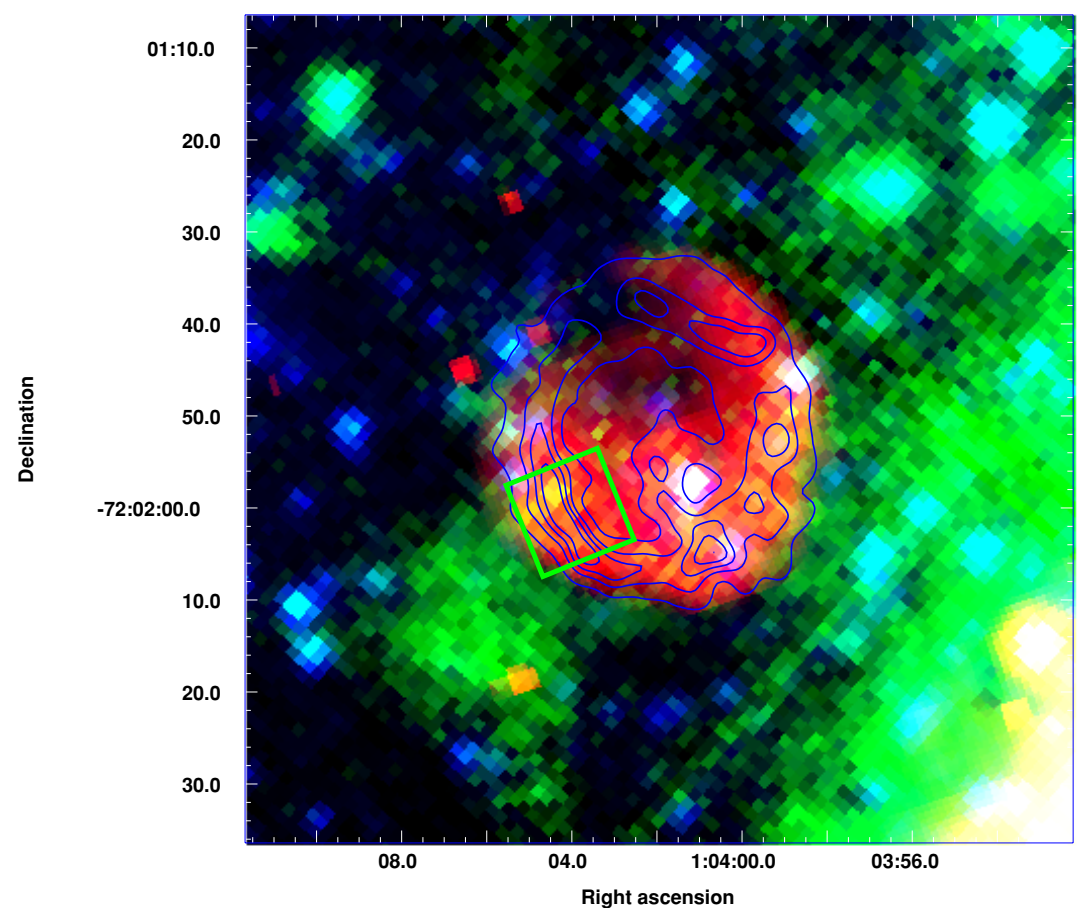

Figure 6. Mosaicked three-color infrared images of E0102. Red: MIPS $24 \mu \mathrm{m}$ (from Stanimirovic et al. 2005); green: IRAC $8 \mu \mathrm{m}$; blue: IRAC 5.8 $\mu \mathrm{m}$ images. The IRAC $8 \mu \mathrm{m}$ flux ranges from 0.15 to $0.2 \mathrm{MJy} \mathrm{sr}^{-1}$. Possible detection of faint $8 \mu \mathrm{m}$ emission (seen in yellow) from the southeastern shell is suggested. The IRS slit coverage is marked as a green box. Note that the remnant is not detected at $5.8 \mu \mathrm{m}$. The bright emission at the bottom right of the image is from N76. X-ray contours (Gaetz et al. 2000) are superposed.
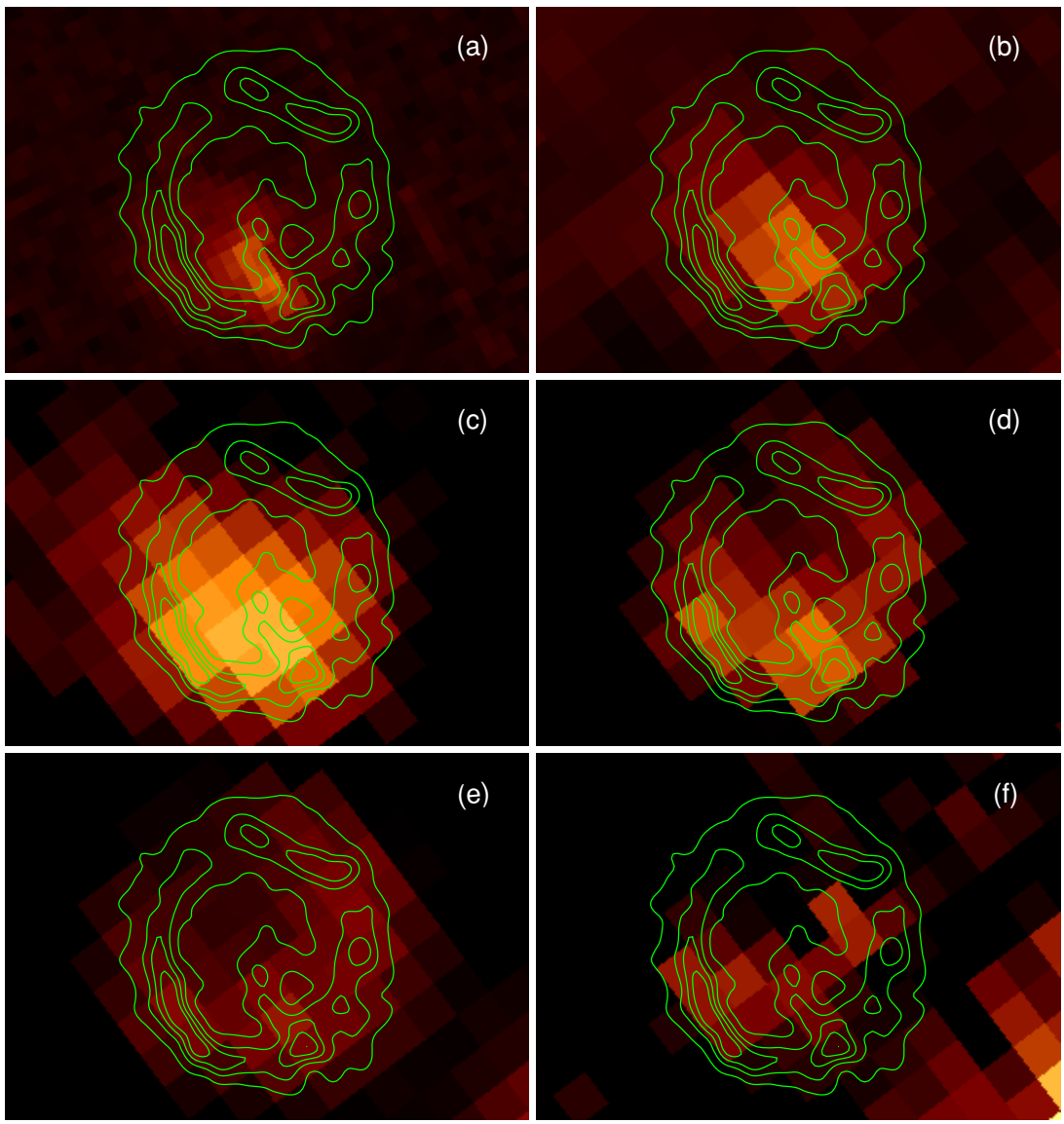

Figure 7. Line map images of E0102: (a) [Ne II] $12.8 \mu \mathrm{m}$, (b) [Ne III] $15.55 \mu \mathrm{m}$, (c) [O IV]+[Fe II] $26 \mu \mathrm{m}$, (d) continuum 16.5-20.5 $\mu \mathrm{m}$, (e) continuum 27-31 $\mu \mathrm{m}$, and (f) [Si II] $34.8 \mu \mathrm{m}$. X-ray contours (Gaetz et al. 2000) are superposed. The spatial resolution of [Ne II] image (from SL) is $\sim 2$ " and of the other images (from LL) is $\sim 5^{\prime \prime} .1$. 

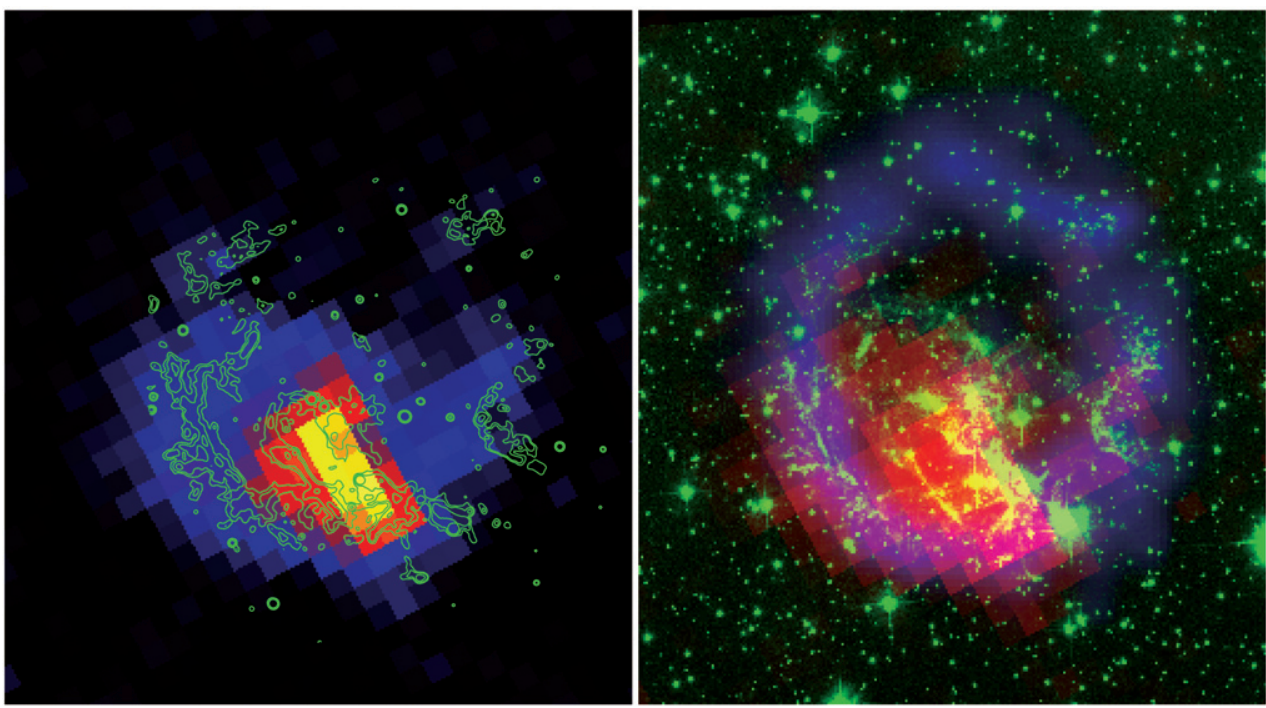

Figure 8. Left: Spitzer [Ne II] intensity map (colors are from yellow, red, and blue in strength of brightness) overlaid with HST [O III] contours. Right: color images of $\mathrm{X}$-ray (blue), optical [O III] (green), and infrared [Ne II] $12.8 \mu \mathrm{m}$ (red) emission.

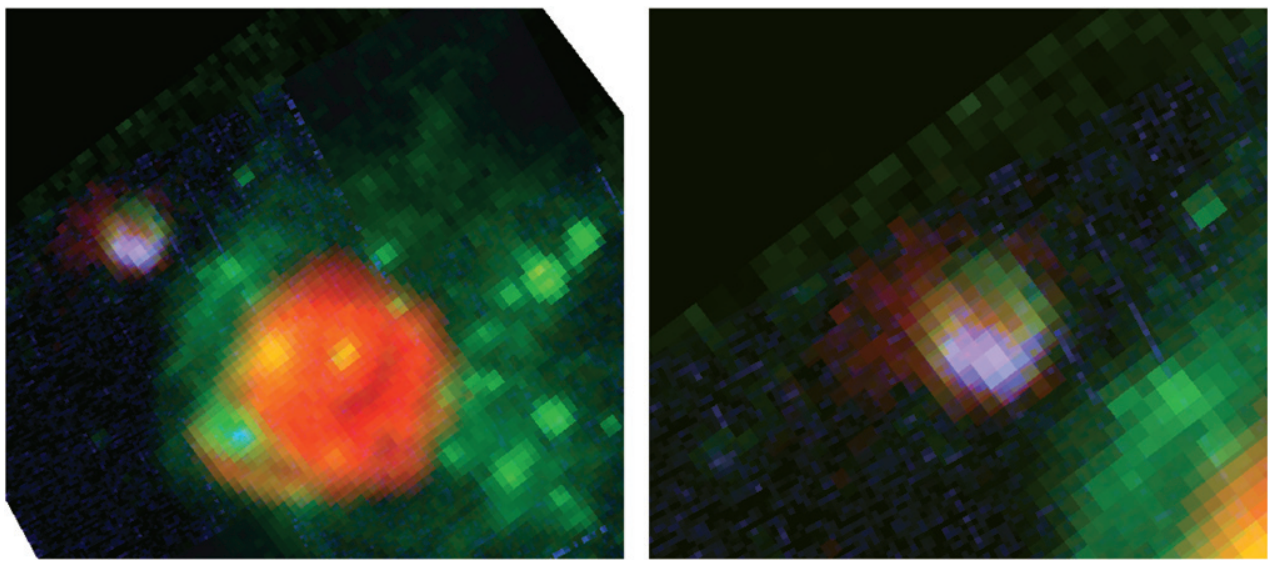

Figure 9. Mosaicked three-color images of (a) E0102 and N76, (b) E0102 only: [Ne II] (blue), 16.5-20.5 $\mu$ m continuum (green) and [O IV] (red). The image on the left is centered on R.A. $=01^{\mathrm{h}} 03^{\mathrm{m}} 45^{\mathrm{s}} .6$ and decl. $=-72^{\circ} 02^{\prime} 02^{\prime \prime} \cdot 3$, and the FOV is 5.2. The image on the right is centered on E0102 and the FOV is 2.7.

of the SNR. This may indicate that the freshly formed dust is correlated not only with infrared ejecta, but also with optical and X-ray ejecta (recall that the infrared continuum shell is still located inside the forward shock so it is less likely due to CSM/ ISM material). Dust forms in the ejecta when the gas cools below a temperature of 1000-2000 K. After the reverse shock encountered the ejecta, it sufficiently heated both gas and dust, which emit the infrared emission lines and continuum, respectively.

\subsection{Doppler-shifted Lines and Maps}

The measured [Ne II] line width of the staring-mode spectrum (southeastern (SE) shell position in Figure 2) is $0.1529 \pm$ $0.0023 \mu \mathrm{m}$ (Table 3). To examine the velocity broadening, we extracted SL1 spectra from three additional positions in E0102 - center, east, and Scenter (see the boxes of Figure 10). The three spectra show different line widths, as shown in Figure 11. The Doppler-shifted line maps in Figure 10, with the locations of the three spectra overlaid show that the central position has both blue and redshifted emission, while the eastern position has blueshifted emission. The properties of the line widths at these positions are summarized in Table 3. At the center, the measured $\sim 0.12 \mu \mathrm{m}$ line width is comparable to those measured for the [Ne II] calibration sources G333.9,
NGC6720, and NGC7293. By contrast, the widths in the center and $\mathrm{E}$ spectra do indicate line broadening.

Fitting one Gaussian component, the line widths correspond to velocity dispersions of $5226 \pm 304 \mathrm{~km} \mathrm{~s}^{-1}, 3579 \pm 54 \mathrm{~km} \mathrm{~s}^{-1}$, and $3433 \pm 64 \mathrm{~km} \mathrm{~s}^{-1}$ for the center, SE shell, and E, respectively. After taking into account the spectral resolution, we can estimate the true velocity dispersion for each position (Table 3). The center, E, and SE shell positions show the true velocity dispersion of $4446_{-361}^{+353}, 2060_{-108}^{+104}$, and $2295_{-83}^{+85} \mathrm{~km} \mathrm{~s}^{-1}$, respectively. The observed velocity shifts and dispersions are comparable to those from optical measurements from -2600 to $3640 \mathrm{~km} \mathrm{~s}^{-1}$ (Tuohy 1983; Blair et al. 2000). The broad-line profile for the center can also be fitted with two Gaussian components. Using a rest wavelength of [Ne II] of $12.8135 \mu \mathrm{m}$, the ejecta material must then be redshifted by $2615 \mathrm{~km} \mathrm{~s}^{-1}$ and blueshifted by $641 \mathrm{~km} \mathrm{~s}^{-1}$. A confirmation with higher spectral resolution data is desirable because of the limited spectral resolution of IRS low-resolution spectra.

\subsection{Ne Line-Flux Ratio}

In the line list in Table 2, the ions [Ne III] and [Ne v] offer pairs of lines which are suitable for line diagnostics in order to constrain densities and temperatures. The measured line- 
Table 3

Properties of Ne Line Broadening

\begin{tabular}{lcccccc}
\hline \hline Location & R.A. & Decl. & Color & Wavelength $(\mu \mathrm{m})$ & FWHM $(\mu \mathrm{m})$ & ${\text { Velocity }\left(\mathrm{km} \mathrm{s}^{-1}\right)}^{\text {Velocity }_{\text {true }}^{\mathrm{a}}\left(\mathrm{km} \mathrm{s}^{-1}\right)}$ \\
\hline Center & $01: 04: 02.05$ & $-72: 01: 53.2$ & Blue & $12.8081 \pm 0.0057$ & $0.2235 \pm 0.0134$ & $5226 \pm 304$ \\
& & & $(1)$ & $12.7861 \pm 0.0031$ & $0.16733 \pm 0.0070$ & $3909 \pm 164\left[641^{\mathrm{b}}\right]$ \\
& & & $(2)$ & $12.9252 \pm 0.0045$ & $0.09391 \pm 0.0068$ & $2196 \pm 1640\left[2615^{\mathrm{b}}\right]$ \\
$E$ & $01: 04: 05.01$ & $-72: 01: 55.6$ & Green & $12.7931 \pm 0.0012$ & $0.1465 \pm 0.0028$ & $3433 \pm 64$ \\
Scenter & $01: 04: 02.5$ & $-72: 01: 59.6$ & Red & $12.8104 \pm 0.0026$ & $0.1172 \pm 0.0060$ & $2746 \pm 140$ \\
SE shell & $01: 04: 04.04$ & $-72: 02: 00.5$ & Figure 3 & $12.8145 \pm 0.0010$ & $0.1529 \pm 0.0023$ & $3579 \pm 54$ \\
\hline
\end{tabular}

Notes.

${ }^{a}$ Velocity true is estimated velocity dispersion after accounting for the instrumental resolution.

b Velocity dispersion shift from the line centroid.

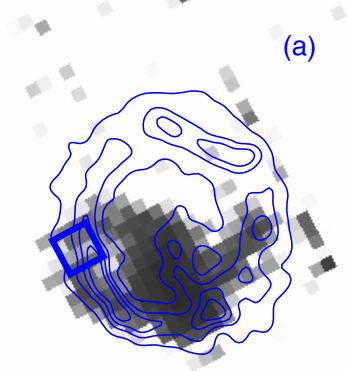

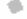

(c)

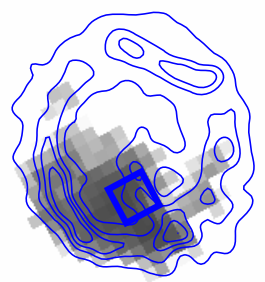

(b)

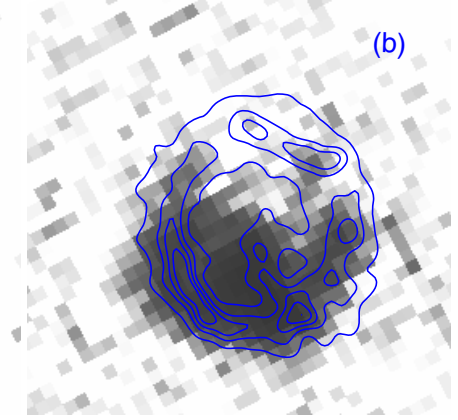

(d)

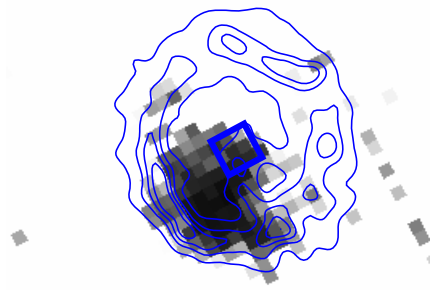

Figure 10. Line maps [Ne II] $(12.8 \mu \mathrm{m})$ showing blue to redshifted wavelength regions: 12.5-12.7 $\mu \mathrm{m}$ (a), 12.7-12.8 $\mu \mathrm{m}$ (b), 12.8-12.9 $\mu \mathrm{m}$ (c), and 12.9$13.1 \mu \mathrm{m}(\mathrm{d})$. The maps are overlaid with the Chandra X-ray contours. The positions of the spectra in Figure 11 are marked as boxes in the maps, (a), (c), and (d) for the locations of "E," "Scenter," and "center," respectively (see Table 3).

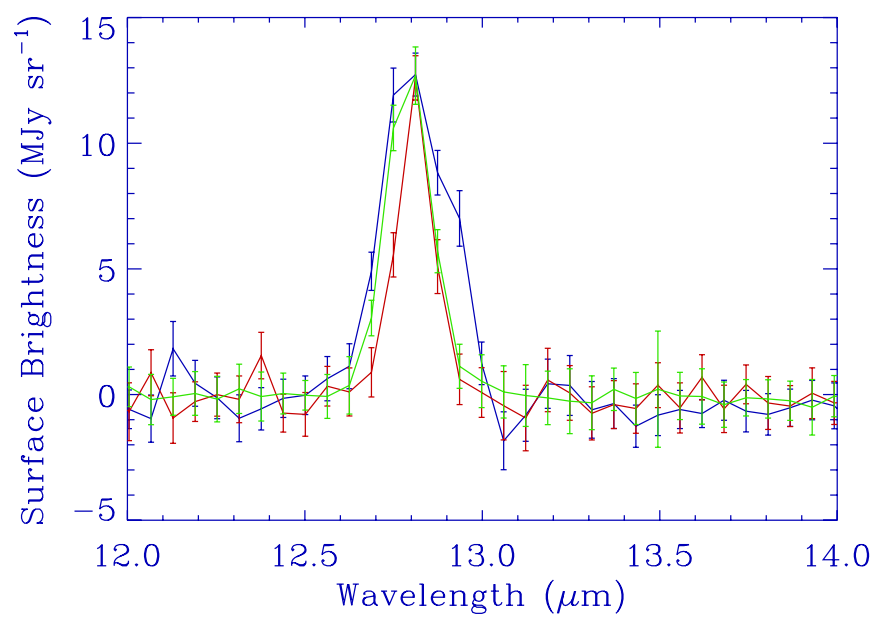

Figure 11. Spectral line profiles of [Ne II] toward three positions (see Table 3) in E0102: center (blue), E (green), and Scenter (red). The line broadening in the center spectrum is consistent with a velocity of $5226 \mathrm{~km} \mathrm{~s}^{-1}$ (see Table 3 for details).

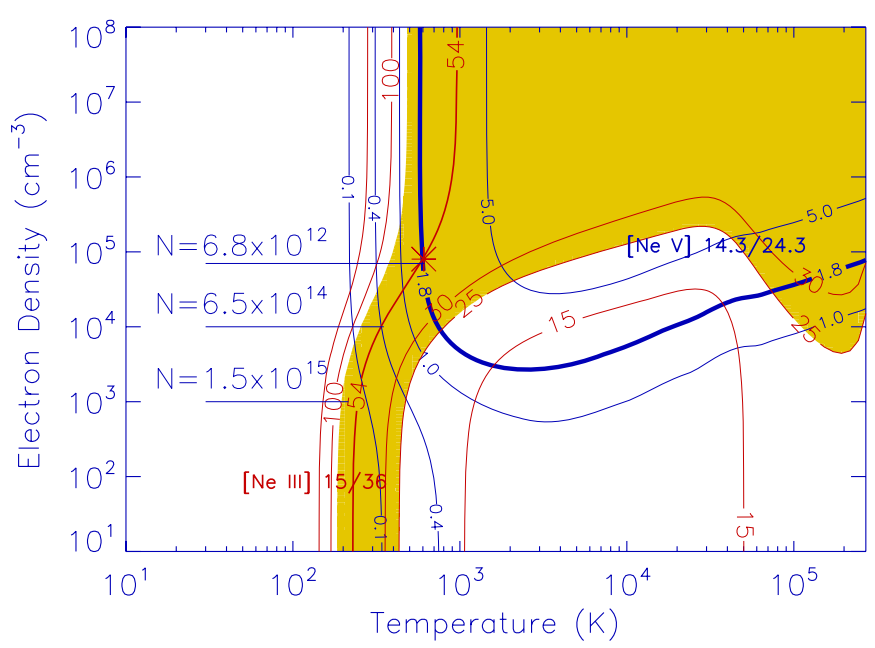

Figure 12. Line diagnostic contours of the ratios of [Ne III] $15 / 35 \mu \mathrm{m}$ (red) and [Ne v] 14.3/24.3 $\mu \mathrm{m}$ (blue). The observed ratios are marked with the thick solid lines for the best value of the ratios $[\mathrm{Ne} \mathrm{III}]=54$ and $[\mathrm{Ne} \mathrm{v}]=1.76$. Note that the density is an electron density. The shaded region shows the range of temperatures and densities allowed by errors for the [Ne III] ratio, whereas the allowed physical conditions of [ $\mathrm{Ne} \mathrm{v}]$ lines are marked with the thick blue contour lines. The contours of [Ne III] 15/36 and [Ne v] 14.3/15.3 intersect at the solution with a temperature of $612 \mathrm{~K}$ and a density of $4 \times 10^{4} \mathrm{~cm}^{-3}$ (marked as an asterisk). The column density of [Ne III] is marked for three different densities (see Table 5).

flux ratios for $[\mathrm{Ne} v] \lambda 14.3 / 24.3 \mu \mathrm{m}$ are $1.76 \pm 0.11$, and for $[\mathrm{Ne}$ III $] \lambda 15.6 / 36 \mu \mathrm{m} 54 \pm 28$ (see Figure 12). The ratio $15.6 / 36 \mu \mathrm{m}$ is assigned an additional $50 \%$ systematic error for the $36 \mu \mathrm{m}$ line intensity, because this line falls on the degrading part of the array. To constrain temperatures and densities, we calculate the line intensities and ratios of $[\mathrm{Ne} \mathrm{v}]$ and [Ne III]. We solve the excitation-rate equations including collisional and radiative processes as a matrix using five energy levels for [Ne v] (see Figure 13) and three levels for [Ne III]. The input atomic data were taken from Griffin \& Badnell (2000) ${ }^{7}$ and include temperature-dependent collisional strengths. The solutions of the [ $\mathrm{Ne} \mathrm{v}$ ] line diagnostics differ between the newer temperature-independent collisional strengths of Griffin \& Badnell (2000) and older values (e.g., Osterbrock 1989), while those of [Ne III] are almost the same. The line diagnostics are shown in Figure 12. The density and temperature jointly obtained from the ratios of $[\mathrm{Ne} \mathrm{V}]$ and $\left[\mathrm{Ne} \mathrm{III]} \mathrm{are} 7 \times 10^{4}(>9000) \mathrm{cm}^{-3}\right.$ and $612_{-200}^{+300} \mathrm{~K}$, when assuming that both $[\mathrm{Ne} \mathrm{III}]$ and $[\mathrm{Ne} \mathrm{V}]$ gas come from the same gas. Such a high density seems to be characteristic of SN ejecta as observed in optical lines; Chevalier

7 See http://www-cfadc.phy.ornl.gov/data_and_codes/aurost/aurost_excit/. 


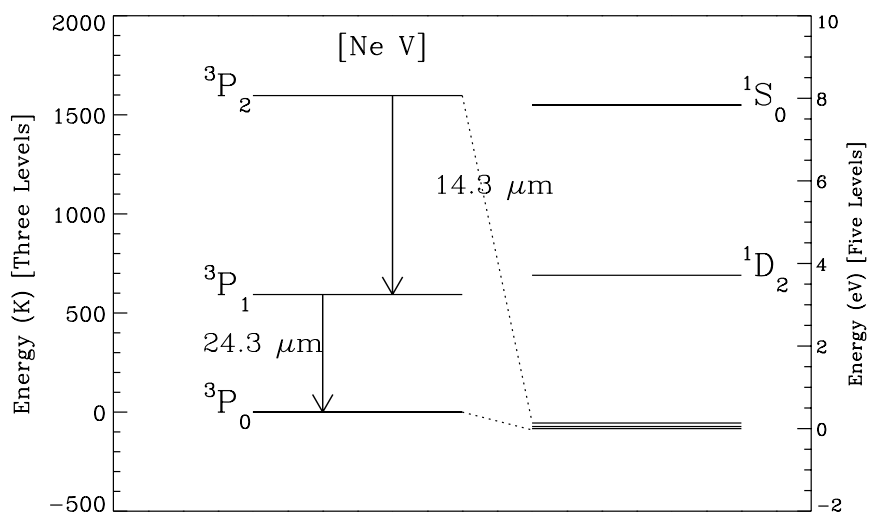

Figure 13. Energy diagram of [ $\mathrm{Ne} v$ ]. Five levels are shown to the right in a unit of $\mathrm{eV}$, with a zoom-in of the three ground-stage levels to the left (with a unit of $\mathrm{K}$ ).

\& Kirshner (1979, 1978), for example, suggested that the optical [S II] line in Cas A originated from ejecta with a density of $10^{5} \mathrm{~cm}^{-3}$.

\section{DISCUSSION}

\subsection{Dust Spectral Fitting and Dust Mass of E0102}

The IRS spectra of E0102 have a prominent dust feature peaking at $18 \mu \mathrm{m}$ which coincides spatially with the emission from the infrared-emitting ejecta. This is clear evidence that dust is forming in the ejecta. To determine the dust composition and mass, we performed spectral fitting to the IRS dust continuum using the deep IRS spectra shown in Figure 3. Note that the wavelength range used for the fits is $5-40 \mu \mathrm{m}$, and that only the continuum emission is used to estimate the dust mass.

First, we estimated the contribution of the synchrotron emission to the infrared spectrum using the radio fluxes and spectral index. The radio flux at $408 \mathrm{MHz}$ is $0.65 \mathrm{Jy}$ for the entire SNR (Amy \& Ball 1993). We measured the radio surface brightness at the IRS position to be $0.58 \mathrm{MJy} \mathrm{sr}^{-1}$ from the $408 \mathrm{MHz}$ image of Amy \& Ball (1993). For a radio spectral index $\alpha=-0.70$ (Amy \& Ball 1993), where $S_{v} \propto v^{\alpha}$, the expected synchrotron fluxes are $1.4 \times 10^{-4}, 3.7 \times 10^{-4}$, and $5.5 \times 10^{-4} \mathrm{MJy} \mathrm{sr}^{-1}$ at 5,20 , and $35 \mu \mathrm{m}$, respectively. This is a very small contribution to the infrared continuum $(<1 \%)$.

The dust continuum was then modeled with the Planck function $B_{v}(T)$ multiplied by the absorption efficiency $\left(Q_{\mathrm{abs}}\right)$ for various dust compositions, with the amplitude and temperature of different components allowed to vary. The fitting technique and mass estimation method is the same as that was used for Cas A (Rho et al. 2008). The spectrum shortward of $10 \mu \mathrm{m}$ is highly uncertain due to background subtraction and instrumental noise. Three dust components are needed to fit the IRS spectrum of E0102.

The $18 \mu \mathrm{m}$ peak dust feature includes silicates, but it cannot be reproduced by the combination of $\mathrm{C}, \mathrm{Mg}$-rich silicates, and $\mathrm{Al}_{2} \mathrm{O}_{3}$ grains that are most likely to be associated with the Neand O-rich ejecta. A component with a feature around $16 \mu \mathrm{m}$ is also needed. Candidates include $\mathrm{MgO}$ and $\mathrm{Si}$. Whereas the $\mathrm{MgO}$ feature calculated using the optical constants of Hofmeister et al. (2003) turns out to be too narrow, amorphous Si, which can condense in the C-rich layer in the primordial SNe II (Nozawa et al. 2003), can successfully reproduce the observed feature.

The rest of the continuum requires either carbon (Model A) or $\mathrm{Al}_{2} \mathrm{O}_{3}$ (Model B) dust, as shown in Figures 14 and 15 . We favor carbon or $\mathrm{Al}_{2} \mathrm{O}_{3}$ over solid $\mathrm{Fe}$ dust, because we expect

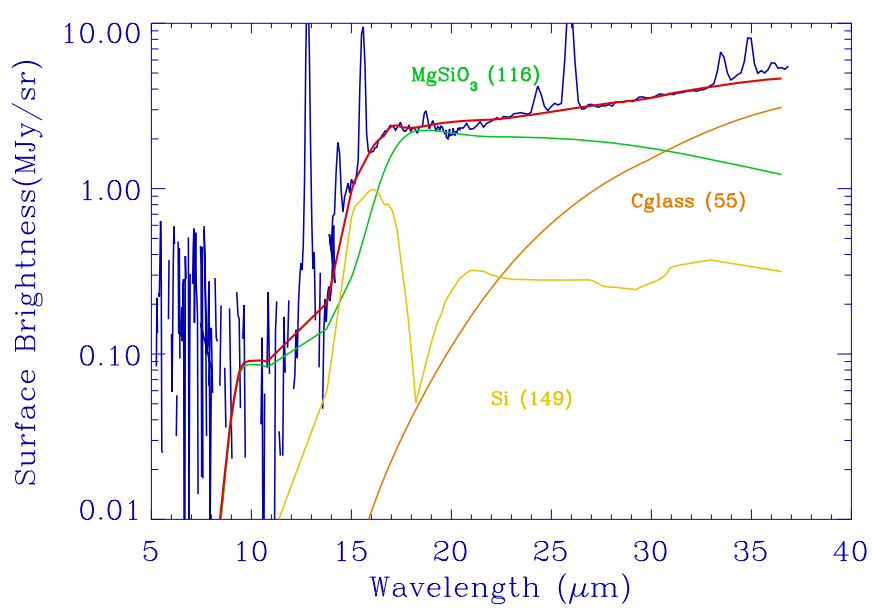

Figure 14. E0102 IRS dust spectrum superposed on a dust fit (Model A in Table 4). The continuum was fitted with dust compositions of $\mathrm{Si}, \mathrm{MgSiO}_{3}$, and carbon. The compositions suggest that the dust forms around carbonburning layers. The data and the total fit are shown in blue and thick red lines, respectively. Contributions from each type of dust are shown with the dust temperatures given in parentheses. The gas lines are also shown in the plot, but they are excluded in the spectral fitting (see Figure 15).

(A color version of this figure is available in the online journal.)

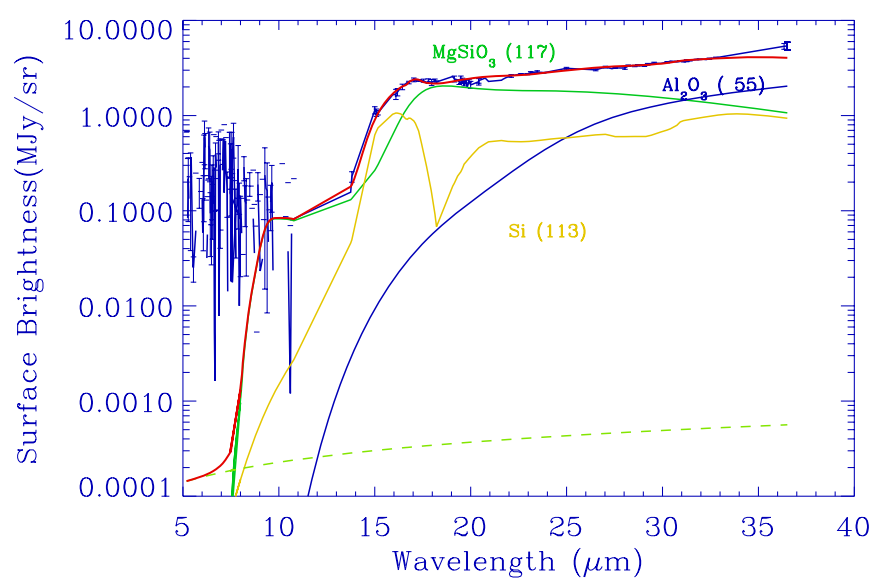

Figure 15. Dust continuum superposed on the dust fit of Model B (Table 4) with the dust compositions of $\mathrm{Si}, \mathrm{MgSiO}_{3}$, and $\mathrm{Al}_{2} \mathrm{O}_{3}$. The synchrotron continuum contribution estimated from the radio fluxes and spectral index is shown by the green dashed line.

(A color version of this figure is available in the online journal.)

carbon or $\mathrm{Al}_{2} \mathrm{O}_{3}$ dust to be present where the $\mathrm{Ne}$ and $\mathrm{O}$ ejecta lines are dominant: $\mathrm{Ne}, \mathrm{Mg}$, and $\mathrm{Al}$ are all carbon-burning nucleosynthesis products (Woosley 1995). $\mathrm{MgSiO}_{3}$ and $\mathrm{Al}_{2} \mathrm{O}_{3}$ are condensed in the ejecta of $\mathrm{Ne}, \mathrm{Mg}$, and $\mathrm{Al}$, which are also carbon-burning products; the $\mathrm{O}$ and $\mathrm{Al}$ are found in the outer layers of ejecta, which is where $\mathrm{Si}$ and carbon dust grains condense. The dust and ejecta compositions seen in E0102 are similar to those of the weak $21 \mu \mathrm{m}$ dust layer in Cas A (Rho et al. 2008). E0102, however, does not have the same silicate dust features seen in Cas A. Also, unlike Cas A, the dust composition in different ejecta layers in E0102 cannot be spatially resolved, at least partly because observations of E0102 suffer from limited angular resolution due to its 18 times greater distance.

The dust compositions, temperatures, and masses obtained from the best fits are summarized in Table 4 . The estimated dust masses within the IRS observed slit are $5.0 \times 10^{-3}$ and $5.5 \times 10^{-3} M_{\odot}$ for Models $\mathrm{A}$ (carbon) and $\mathrm{B}\left(\mathrm{Al}_{2} \mathrm{O}_{3}\right)$, respectively. To determine the sensitivity to dust temperature, in Model $\mathrm{C}$ we performed the fit allowing the temperature of $\mathrm{Al}_{2} \mathrm{O}_{3}$ 
Table 4

Properties of Freshly Formed Dust in E0102

\begin{tabular}{|c|c|c|c|c|}
\hline Model & $\Delta \chi^{2}\left(=\chi^{2} / \text { dof }\right)^{\mathrm{a}}$ & Dust Compositions (Temperature $(\mathrm{K})$, Mass $\left.\left(M_{\odot}\right)\right)^{\mathrm{b}}$ & Mass $\left(M_{\odot}\right)^{\mathrm{c}}$ & Total Mass $\left(M_{\odot}\right)^{\mathrm{c}}$ \\
\hline A & $2.41(\sim 398 / 165)$ & $\mathrm{MgSiO}_{\mathbf{3}}(\mathbf{1 1 6}, \mathbf{3 . 8 0 \mathrm { E }}-5), \mathrm{Si}(\mathbf{1 5 0}, \mathbf{6 . 2 0 E}-5), C(55,5.00 E-3)$ & $5.12 \times 10^{-3 \mathrm{~d}}$ & $0.015^{\mathrm{d}}$ \\
\hline $\mathrm{C}$ & $2.18(\sim 438 / 201)$ & $\mathrm{MgSiO}_{3}(108,6.25 \mathrm{E}-5), \mathrm{Si}(142,9.21 \mathrm{E}-5), \mathrm{Al}_{2} \mathrm{O}_{3}(41,0.03)^{\mathrm{e}}$ & $0.03^{\mathrm{e}}$ & $0.09^{\mathrm{e}}$ \\
\hline & & $\mathrm{Al}_{2} \mathrm{O}_{3}(60,3.33 \mathrm{eE}-4), \mathrm{C}(55,4.350 \mathrm{E}-3), \mathrm{Mg}_{2} \mathrm{SiO}_{4}(100,1.10 \mathrm{E}-5)$ & $4.80 \times 10^{-3 \mathrm{f}}$ & $0.014^{\mathrm{f}}$ \\
\hline
\end{tabular}

Notes.

a $\Delta \chi^{2}$ is the reduced $\chi^{2}$ and "dof" is the degrees of freedom.

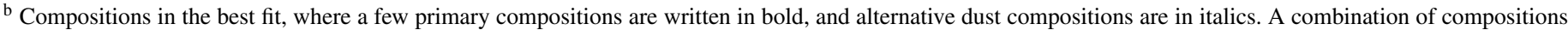
are in a regular font.

${ }^{c}$ Mass is the mass within the IRS slit and the total mass is the mass estimated over the entire SNR (see the text for details).

$\mathrm{d}$ The errors are on order of $10 \%-20 \%$.

e The errors could not be constraint.

f The errors are on order of $20 \%-40 \%$.

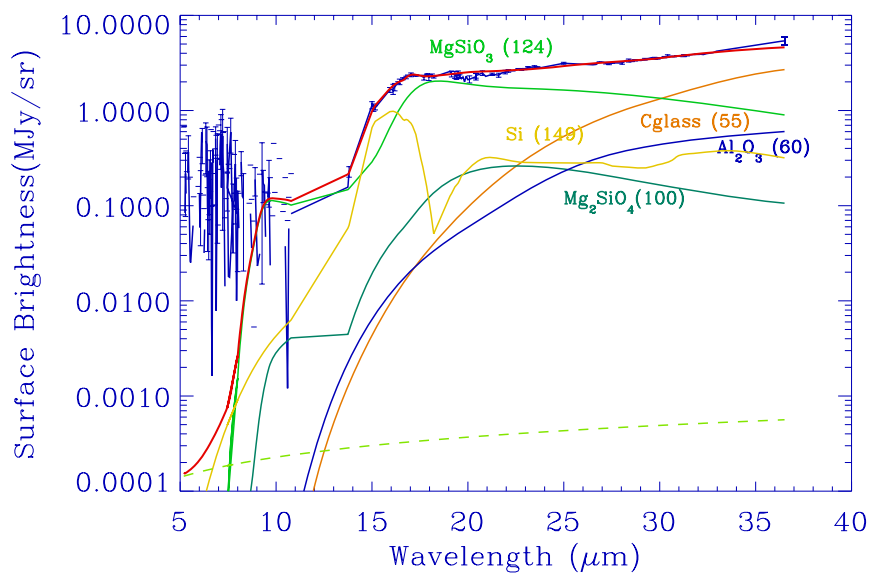

Figure 16. Dust continuum superposed on a combination of dust models of $\mathrm{Si}$, $\mathrm{MgSiO}_{3}, \mathrm{Al}_{2} \mathrm{O}_{3}, \mathrm{Mg}_{2} \mathrm{SiO}_{4}$, and carbon.

(A color version of this figure is available in the online journal.)

to vary independently. Model $\mathrm{C}$ produced a low temperature $(<45 \mathrm{~K})$ that cannot be constrained by our spectra, which lack long-wavelength data; therefore, we do not consider Model C further and fixed the temperature of $\mathrm{Al}_{2} \mathrm{O}_{3}$ in Model $\mathrm{B}$ to that of carbon in Model A. To explore the effect of including many minerals, in Model D we performed a fit including all the most common dust compositions (i.e., $\mathrm{MgSiO}_{3}, \mathrm{Si}, \mathrm{Al}_{2} \mathrm{O}_{3}$, carbon, and $\mathrm{Mg}_{2} \mathrm{SiO}_{4}$; see, e.g., T01, N03). The mass is relatively well constrained by the observations (within $20 \%-40 \%$ ). The fit of Model D is shown in Figure 16. However, the reduced $\chi^{2} \sim 2$ is still relatively high, largely due to the residuals near where the gas lines were subtracted. (Note that some of lines show kinematic broadening.)

To estimate the total dust mass, we correct the observed mass (within the high-sensitivity spectral slit) for the fraction of the SNR covered by the IRS slit using a continuum map (between 21 and $23.5 \mu \mathrm{m}$ ) generated from the lower sensitivity spectral cube. This approach is justified because the spectra from the cube did not show significant variation across the SNR. The correction corresponding to the total flux of the entire SNR relative to the flux of the regions covered by the IRS slit is a factor of 2.9. The total dust masses for the entire SNR are then $0.015,0.007$, and $0.014 M_{\odot}$ for Models $\mathrm{A}, \mathrm{B}$, and D, respectively. We favor Models A or D over Models B or C, because the temperature of $\mathrm{Al}_{2} \mathrm{O}_{3}$ could not be constrained.

$\mathrm{MgSiO}_{3}, \mathrm{Al}_{2} \mathrm{O}_{3}$, and carbon are major dust species predicted to be produced in the ejecta of SNe by both Todini \& Ferrara
(2001) and Nozawa et al. (2003). Note that presolar $\mathrm{Al}_{2} \mathrm{O}_{3}$ grains from meteorites have been inferred to be among the most abundant isotopically enriched materials ejected by Type II SNe (Clayton \& Nittler 2004). In terms of amorphous $\mathrm{Mg}_{2} \mathrm{SiO}_{4}$ versus $\mathrm{MgSiO}_{3}$, the YSNRs Cas A (Rho et al. 2008) and SN 2006jc (Nozawa et al. 2008) also show a larger mass of $\mathrm{MgSiO}_{3}$ than $\mathrm{Mg}_{2} \mathrm{SiO}_{4}$, whereas Nozawa et al. (2003) and Todini \& Ferrara (2001) predict the reverse. This may suggest that the chemical network favors $\mathrm{MgSiO}_{3}$ over $\mathrm{Mg}_{2} \mathrm{SiO}_{4}$.

\subsection{Ionization States of $\mathrm{Ne}$}

Column densities for [ $\mathrm{Ne} \mathrm{III}]$ and $[\mathrm{Ne} \mathrm{V}]$ can be determined from the excitation matrix given by

$$
I(i, j)=\left(\frac{1}{4 \pi}\right) N_{i} A(i, j) h v_{i j},
$$

where $I(i, j)$ is a line intensity between levels $i$ and $j, N_{i}$ is a column density in the level $i, A(i, j)$ is the spontaneous radiation transition rate, $N_{i}$ is a column density in the level $i$, and $v_{i j}$ is the energy difference between $i$ and $j$ levels. For level $i$, the matrix to solve the column density $N_{i}$ is given by

$$
\begin{gathered}
\Sigma_{j<i} N_{i} A_{i j}+\Sigma_{j} N_{i} n \gamma_{i j}=\Sigma_{j<i} N_{j} A_{j i}+\Sigma_{j} N_{j} n \gamma_{j i}, \\
\frac{\gamma(j, i)}{\gamma(i, j)}=\frac{g_{i}}{g_{j}} \exp ^{-h v_{i j} / k T},
\end{gathered}
$$

where $n$ is the density, $T$ is the temperature, and $\gamma(i, j)$ and $\gamma(j, i)$ are the collisional transition and deexcitation rates. The total column density of $N=\Sigma_{i} N_{i}$ is obtained by solving the matrix. For example, the total column density of [ $\mathrm{Ne} \mathrm{v}]$ is the sum of all five levels as shown in Figure 13. For a temperature of $612 \mathrm{~K}$ and a density of $7 \times 10^{4} \mathrm{~cm}^{-3}$, the column densities for [Ne III] and [Ne v] are $6.8 \times 10^{12}$ and $5.9 \times 10^{11} \mathrm{~cm}^{-2}$, respectively. We estimated the column density for a few sets of densities and temperatures implied by the observed [Ne III] ratio as shown in Figure 12, and found that the column density changes rapidly depending on the electron density as listed in Table 5. We also solved the excitation-rate equation using two energy levels for Ne II. The observed line brightness of [Ne II] can be used to estimate the ion column density to be $2.7 \times 10^{13} \mathrm{~cm}^{-2}$. The estimated column densities of $\mathrm{Ne}$ are summarized in Table 5.

The nucleosynthetic yields of $\mathrm{Ne}$ trace the progenitor mass. In order to obtain the $\mathrm{Ne}$ ejecta mass, we must estimate the 
Table 5

Summary of Ne Column Density and Mass Estimates

\begin{tabular}{|c|c|c|c|c|}
\hline \multirow[t]{2}{*}{ Ne Ionization State } & \multicolumn{3}{|c|}{ From Excitation-rate Equation } & \multirow[t]{2}{*}{ From Shock Model ${ }^{\mathrm{a}}$} \\
\hline & Model $\mathrm{A}^{\mathrm{b}}$ & Model $\mathrm{B}^{\mathrm{b}}$ & Model $C^{b}$ & \\
\hline$[\mathrm{Ne} I]\left(\mathrm{cm}^{-2}\right)$ & $2.4 \times 10^{15}$ & & & $1.5 \times 10^{15}$ \\
\hline$[\mathrm{Ne} \mathrm{II}]\left(\mathrm{cm}^{-2}\right)$ & $2.7 \times 10^{13}$ & $4.4 \times 10^{14}$ & $2.4 \times 10^{16}$ & $7.8 \times 10^{15}$ \\
\hline$[\mathrm{Ne} \mathrm{III}]\left(\mathrm{cm}^{-2}\right)$ & $6.8 \times 10^{12}$ & $6.5 \times 10^{14}$ & $1.5 \times 10^{15}$ & $1.2 \times 10^{15}$ \\
\hline$[\mathrm{Ne}$ IV $]\left(\mathrm{cm}^{-2}\right)$ & $9.6 \times 10^{11}$ & $\ldots$ & $\ldots$ & $8.3 \times 10^{13}$ \\
\hline$[\mathrm{Ne} \mathrm{v}]\left(\mathrm{cm}^{-2}\right)$ & $5.9 \times 10^{11}$ & $6.0 \times 10^{11}$ & $6.0 \times 10^{11}$ & $1.1 \times 10^{14}$ \\
\hline Ne I-V total $\left(\mathrm{cm}^{-2}\right)$ & $7.8 \times 10^{16}$ & $1.1 \times 10^{15}$ & $2.6 \times 10^{16}$ & $4.0 \times 10^{16}$ \\
\hline Ne mass ${ }^{\mathrm{c}}\left(M_{\odot}\right)$ in IRS slit & 0.22 & 0.003 & 0.07 & 0.014 \\
\hline Total Ne mass ${ }^{\mathrm{c}}\left(M_{\odot}\right)$ & 0.63 & 0.01 & 0.21 & 0.042 \\
\hline
\end{tabular}

Notes.

a The best estimates (in bold) are from our shock model.

${ }^{\mathrm{b}}$ Model A: $n_{e}=7 \times 10^{4} \mathrm{~cm}^{-3}, T=612 \mathrm{~K}$; Model B: $n_{e}=1 \times 10^{4} \mathrm{~cm}^{-3}, T=350 \mathrm{~K}$; Model C: $n_{e}=1 \times 10^{3} \mathrm{~cm}-3, T=215 \mathrm{~K}$. See the text and Figure 12 for details.

${ }^{\mathrm{c}} \mathrm{Ne}$ mass is the mass within the IRS slit and the total Ne mass is the mass estimated over the entire SNR.

column densities of the remaining ionization stages of $\mathrm{Ne}$. First, we obtain the column densities when we assume local thermodynamic equilibrium, which is described below in this section. Later, we use shock models to estimate the column densities of ionization states as described in Section 4.3.

The Saha equation is given by

$$
\begin{aligned}
\ln \left(\frac{N_{r+1}}{N_{r}} N_{e}\right)= & \ln \left[\frac{U_{r+1}}{U_{r}} \frac{2\left(2 \pi m_{p} k T\right)^{3 / 2}}{h^{3}} \exp \left(\frac{-\chi}{k T}\right)\right] \\
& \sim \ln \left(\frac{U_{r+1}}{U_{r}}\right)+36.11413+\frac{3}{2} \ln T \\
& -11602.81 \frac{I_{\mathrm{eV}}}{T}
\end{aligned}
$$

where $N$ is the number density of atoms in the $r$ (or $r+1$ )th stage of ionization, $U$ is the partition function depending on the temperature, $m_{p}$ is the mass of the proton, $T$ is the temperature in Kelvin, and $\chi\left(I_{\mathrm{eV}}\right)$ is the ionization potential for $r$ to $(r+1)$ (in units of eV).

We estimated the column densities of [Ne I] and [Ne IV] using the Saha equation repeatedly as follows. First, we obtained a gas temperature of $5667 \mathrm{~K}$ by using the column densities of [Ne II] and [Ne III]. While this is a much higher temperature than obtained from the [Ne III] and [Nev] line diagnostics, it is consistent with the error range for the line ratios (see Figure 12). Second, we estimated the neutral [Ne I] column density by using the column density of [Ne II] and a temperature of $5667 \mathrm{~K}$. Third, we estimated the [Ne v] column density by using the temperature of $5667 \mathrm{~K}$ and the Saha equation, and found that the inferred [ $\mathrm{Ne} \mathrm{v}$ ] column density was much smaller than the observed value of $5.8 \times 10^{11} \mathrm{~cm}^{-2}$. This indicates that most of the observed column density of [ $\mathrm{Ne} \mathrm{v}]$ comes from a gas with a temperature different from $5667 \mathrm{~K}$. An additional component with a temperature of $9100 \mathrm{~K}$ is required to fit the observed [Ne III] and [Ne v] column densities. The [Ne IV] column density is then obtained by interpolating between [Ne III] and $[\mathrm{Ne} \mathrm{v}$ ]. Figure 17 shows the column density for each ion of $\mathrm{Ne}$ from the two-temperature components derived here. Column densities for the remaining, more ionized $\mathrm{Ne}$ can be calculated for these two temperatures, and can be seen to fall rapidly with increasing ionization. We also estimated the column density of [Ne III] for $\left(n_{e}, \mathrm{~T}\right)=\left(10^{4} \mathrm{~cm}^{-3}, 350 \mathrm{~K}\right)$ and $\left(10^{3} \mathrm{~cm}^{-3}, 215 \mathrm{~K}\right)$. We find that the column density varies depending on density, as summarized in Table 5 and Figure 12, and that it does not

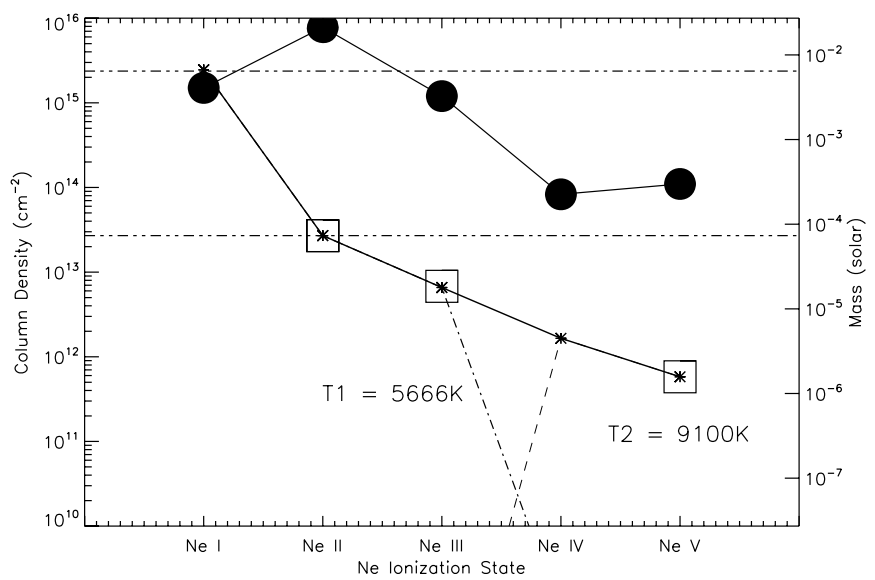

Figure 17. Column density for each Ne ionization state. The column densities are derived from collisional ionization (squares) and from shock models (filled dots). The low- (5667 K, dash-dotted line) and high- (9300 K, dashed line) temperature components are marked with the sum (solid line) of the twotemperature components. The corresponding Ne mass is shown in the right side of $y$-axis. The horizontal lines (dash-three-dotted line) are [Ne I] and [Ne II] column densities and masses.

vary much with temperature. A lower electron density yields a higher column density.

\subsection{Shock Model and Neon Mass}

We have calculated radiative shock models for shocks in the velocity range of $50-500 \mathrm{~km} \mathrm{~s}^{-1}$. These models use many of the same assumptions used by Blair et al. (2000). In particular, we use the same elemental abundances $(\mathrm{H}=$ $12.00, \mathrm{O}=16.00, \mathrm{Ne}=15.50, \mathrm{C}=14.50, \mathrm{Mg}=13.90$ ) and follow their approach of using a single ram pressure, $n_{0} v_{s}^{2}=16 \mathrm{~cm}^{-3} 100 \mathrm{~km} \mathrm{~s}^{-1}$, for each shock speed in the standard case. We also follow Blair et al. (2000) in using a magnetic field strength of $B_{0}=4 \sqrt{n_{0}} \mu \mathrm{G}$ for our standard case. Blair et al. (2000) use the results of a range of shock models with shock velocities ranging from $30-500 \mathrm{~km} \mathrm{~s}^{-1}$, weighting the emission by the fraction of the shock area that is assigned (by assumption) to each shock speed. We take the simpler approach of finding the best-fitting single shock speed. In addition to the standard cases, we have calculated models with ram pressures that are larger by a factor of $1.5,2$, and 3 and with smaller magnetic field strengths, down by a factor of 2 . The model calculations assume one-dimensional (plane parallel) steady 


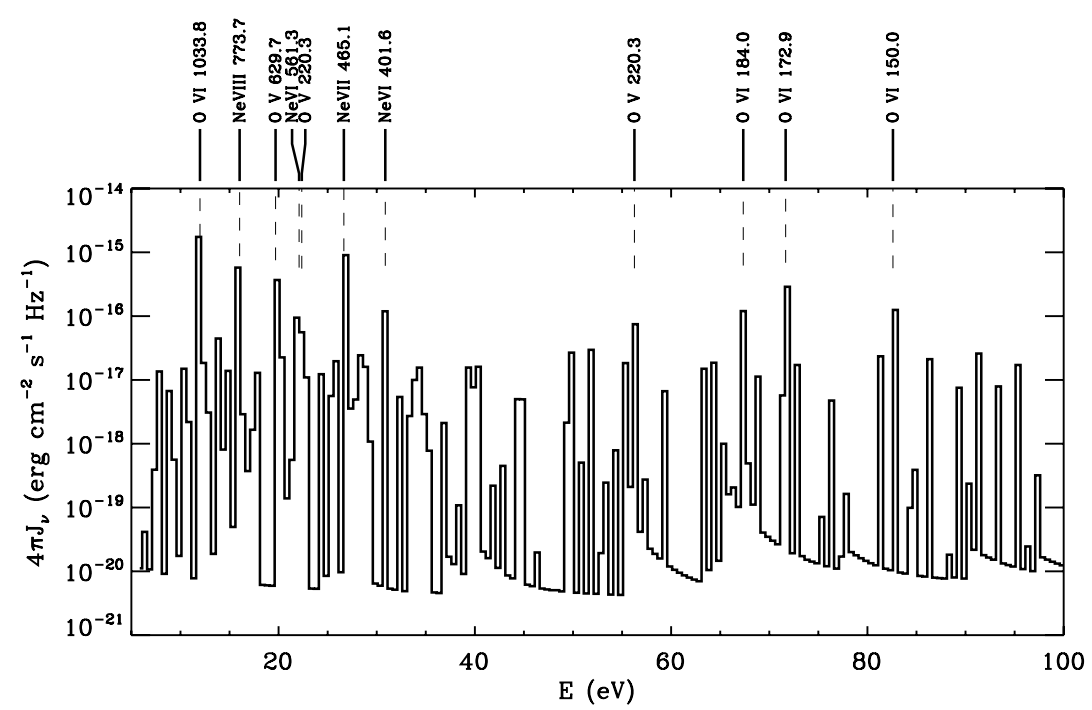

Figure 18. Ionizing radiation generated in the shock and incident on the gas in the photoionization zone for the case $V_{s} \sim 200 \mathrm{~km} \mathrm{~s}^{-1}$ and 3 times the Blair et al. (2000) ram pressure. The spectrum is clearly line dominated and many of the lines are from $\mathrm{O}$ and Ne which have highly enhanced abundances in the ejecta.

flow and include non-equilibrium ionization, radiative cooling and magnetic pressure, $B \propto n$. We also include photoionization from radiation generated in upstream gas and the accompanying heating. Since the region of strong ionizing radiation emission is mainly in the hot, ionized portion of the shock, while the region of substantial photoionization is downstream, this approach is satisfactory. In fact photoionization and heating are negligible within the X-ray/EUV-emitting zones where $T>10^{4} \mathrm{~K}$. These calculations make use of a revised version of the Raymond \& Smith (1977) plasma emission code for the calculation of the hot, ionized postshock zone and the cooling zone in which the gas temperature drops sharply to $\lesssim 1000 \mathrm{~K}$.

For the calculation of the photoionized zone, we use the Cloudy code (version 07.02.02 of the code last described by Ferland et al. 1998) with the incident radiation field taken to be the ionizing radiation field generated in the hot, collisionally ionized and cooling zones (as calculated using the $R \& S$ code). We show an example of the ionizing radiation field in Figure 18. Cloudy assumes thermal and ionization equilibrium (in contrast to our calculations using the R\&S code), but this should not be a very bad assumption for the cool $(T \sim 400-1000 \mathrm{~K})$, dense gas of the photoionized zone. The intensity of the ionizing radiation field depends on the geometrical factor, $R_{\max }$, related to the ratio of the lateral extent of the shock front to its thickness (see, e.g., Raymond 1979). We have generally used $R_{\max }=1$ in our calculations, but found the best agreement for a model with $R_{\max }=0.7$ indicating small shock fronts as might be indicative of shocks propagating into clumps elongated in the radial direction. Figure 19 illustrates the $\mathrm{Ne}$ ionization and temperature for the two parts of the calculation for one model.

As illustrated in the figure, [Ne v] emission is entirely from the ionization and cooling zone, while [Ne II] and [Ne III] emission comes primarily from the low temperature, $T \sim 600 \mathrm{~K}$, photoionization zone. The electron density and temperature derived in Figure 12 from the intersection of the [Ne III] and [Ne v] contours implicitly assumes a uniform temperature and density for the $[\mathrm{Ne} \mathrm{III}]$ and $[\mathrm{Ne} v]$ emitting gas. Since the emission comes from different regions with different temperatures and electron densities, these diagnostics do not apply to emission from the shock.

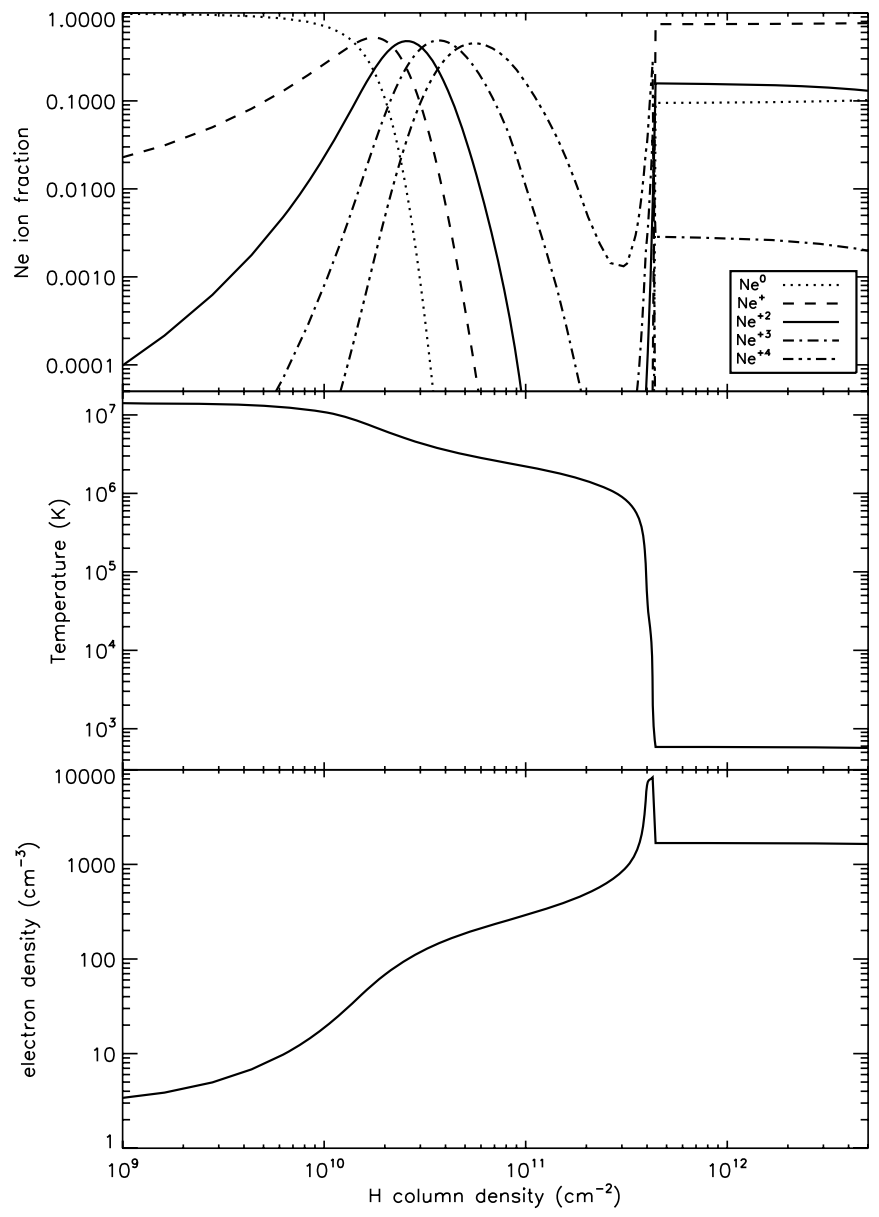

Figure 19. Neon ionization (above) and gas temperature (below) in the shock for our model with shock speed $200 \mathrm{~km} \mathrm{~s}^{-1}$ and ram pressure 3 times the Blair et al. (2000) assumed value. The ionization of Ne clearly shows the lag in ionization and recombination in the ionization $\left(T \geqslant 10^{5} \mathrm{~K}\right)$ and cooling $\left(10^{3} \mathrm{~K} \leqslant T \leqslant 10^{5} \mathrm{~K}\right)$ zones. The region on the right, where $T \sim 550 \mathrm{~K}$, is photoionized and assumed to be in thermal and ionization equilibrium. The $\mathrm{Ne}^{4+}$ ion fraction is very low in the photoionized zone and so the observed [Ne v] $14.3 \mu \mathrm{m}$ and $24.3 \mu \mathrm{m}$ emission come from the cooling zone (an electron density of $10^{3}-10^{4} \mathrm{~cm}^{-3}$ ) whereas most of the [Ne II] and [Ne III] emission comes from the photoionized zone. 


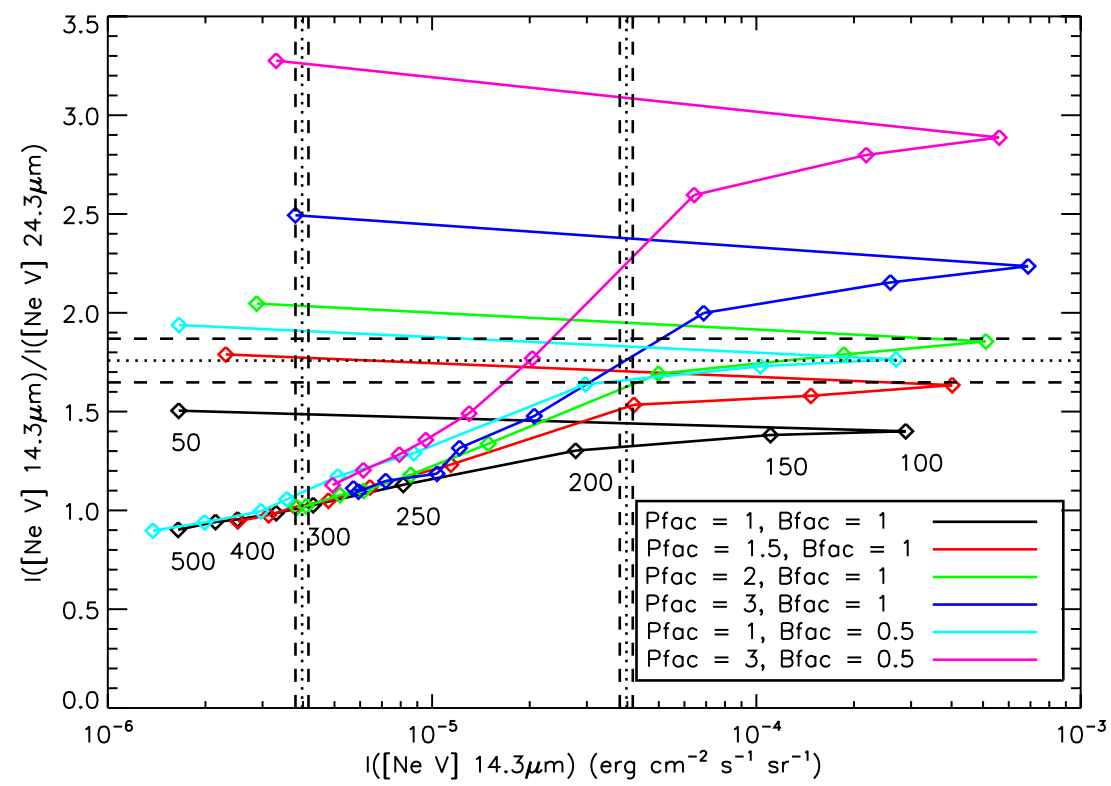

Figure 20. Predicted [ $\mathrm{Ne} \mathrm{v}$ ] line brightness and the ratio of $14.3 / 24.3 \mu \mathrm{m}$ lines depending on shock model parameters of shock velocity (indicated as numbers for the standard case runs), ram pressure $(P)$ and magnetic fields $(B)$. Pfac and $B$ fac indicate the ratio of the value relative to the Blair et al. (2000) values for the ram pressure and magnetic field. The observed Ne v lines are best fit by two shock models of ( $P$ fac $=3, B$ fac $=1$; blue line $)$ and $(P$ fac $=1, B$ fac $=0.5$; light blue line $)$ with a shock velocity of $200-300 \mathrm{~km} \mathrm{~s}^{-1}$. The horizontal dotted line indicates the observed emission line ratio and the dashed lines show the formal $1-\sigma$ errors. The leftmost vertical lines indicate the $14.3 \mu \mathrm{m}$ surface brightness assuming that emission fills the FOV. If the unresolved emission regions only have a filling factor of 0.13 then the true surface brightness is that indicated by the vertical lines to the right. Our "best" model assumes that is the case.

Under the assumption that the $[\mathrm{Ne} \mathrm{III}]$ and $[\mathrm{NeV}]$ emission comes from the same gas, we calculate the column densities of $\mathrm{Ne}$ I, Ne II, and Ne III to be $7 \times 10^{14}, 3.5 \times 10^{15}$, $6.5 \times 10^{14} \mathrm{~cm}^{-2}$, respectively, and the total Ne column density to be $7.9 \times 10^{16} \mathrm{~cm}^{-2}$. To use the results of our shock models we need to limit the extent of the photoionized zone. We use the total [Ne II] $12.8 \mu \mathrm{m}$ emission to set the depth of the photoionized zone. The total [Ne v] emission is naturally limited by the size of the postshock ionization and cooling zones. We find, however, that we are unable to match both the total surface brightness of [Ne v] $14.3 \mu \mathrm{m}$ emission and the ratio of $14.3 \mu \mathrm{m} / 24.3 \mu \mathrm{m}$ emission at the same time when the surface brightness is determined as in Table 2. Matching the line ratio requires lower shock speeds which in turn leads to more flux.

This problem can be overcome if the emission is clumped on scales unresolved by Spitzer. Table 2 uses the default angular extraction size $\left(10^{\prime \prime} \times 20^{\prime \prime}\right.$ for LL) to estimate the surface brightness, because the SNR structures were not resolved. However, we know from the other YSNRs that the angular size of ejecta-emitting regions are generally much smaller than the implied $\sim 3 \mathrm{pc}$. Therefore, we estimate the angular size (to get the solid angle) using a $\mathrm{Ne}$ image of Cas A (Rho et al. 2008). From this we estimate the filling factor of the $\mathrm{Ne}$ emission over the $20^{\prime \prime} \times 10^{\prime \prime}$ field of view (FOV) to be $\sim 0.13$ in surface area. The [Nev] $14.3 \mu \mathrm{m}$ surface brightness of $3.974 \times 10^{-6} \mathrm{erg} \mathrm{s}^{-1} \mathrm{~cm}^{-2} \mathrm{sr}^{-1}$ thus becomes $3.057( \pm 1) \times 10^{-5} \mathrm{erg} \mathrm{s}^{-1} \mathrm{~cm}^{-2} \mathrm{sr}^{-1}$ after the correction, where the errors are estimated using experiments with different surface brightness contours (the filling factor ranging from 0.1 to 0.2 ).

With the correction for the filling factor we find that we can match both the [Ne v] line ratio and fluxes for shock velocities of $\sim 200 \mathrm{~km} \mathrm{~s}^{-1}$, though a better match is achieved for models with a higher, approximately a factor of 3 , ram pressure than the standard Blair et al. (2000) value, as shown in Figure 20. Using the filling factor correction and the best-fitting model for the $[\mathrm{Ne}$ II] and $[\mathrm{Ne} \mathrm{v}]$ emission, we find the column densities of [Ne I], [Ne II], and [Ne III] to be $1.5 \times 10^{15}, 7.8 \times 10^{15}$, and $1.2 \times 10^{15} \mathrm{~cm}^{-2}$, respectively, and the total Ne column density of $4.0 \times 10^{16} \mathrm{~cm}^{-2}$. The column densities from different ionization states of $\mathrm{Ne}$ are summarized in Table 5.

\subsection{Ne Ejecta Mass}

From the column densities for each ion, we can readily calculate the Ne mass by summing over all the ions as

$$
M=\Sigma_{i} N_{i} m_{\mathrm{Ne}} d^{2} \Omega
$$

where $M$ is the total mass, $N_{i}$ is the column density of ion $i, m_{\mathrm{Ne}}$ is the mass of $\mathrm{Ne}, d$ is the distance, and $\Omega$ is the emitting area. In our calculation, we used a wavelength-dependent $\Omega$ which is equivalent to the line extraction width (the approximate area of the LL extraction region is $\left.10^{\prime \prime} \times 20^{\prime \prime}\right)$. The mass corresponding to the column densities for each ion is also shown in Figure 17. The total derived Ne mass (within the IRS slit) inferred from excitation and Saha equations is $0.22 M_{\odot}$ for $n_{e}=7 \times 10^{4} \mathrm{~cm}^{-3}$ and $T=612 \mathrm{~K}$, and $0.07 M_{\odot}$ for $n_{e}=1 \times 10^{3} \mathrm{~cm}^{-3}$ and $T=215 \mathrm{~K}$, respectively. For the shock model, we find a total mass in $\mathrm{Ne}$ of $0.014 M_{\odot}$ which supercedes the derived values.

This is the mass enclosed by the IRS slit only, which is just a portion of the entire SNR. We make the correction for the entire SNR using the [Ne III] map at $15.5 \mu \mathrm{m}$. The total flux is 3.03 times larger than that covered by the slit, so we obtain a total $\mathrm{Ne}$ mass (for all ions including neutral $\mathrm{Ne}$ ) in E0102 of $0.042 M_{\odot}$ from the shock model.

We compared our derived $\mathrm{Ne}$ mass of E0102 with that of SN2005af at an epoch 214 days after the explosion as a rough consistency check. The Ne mass implied from [Ne II] line of SN2005af is $2.2 \times 10^{-3} M_{\odot}$ (Kotak et al. 2006). We determine the [Ne II] mass in E0102 to be $3 \times 10^{-3} M_{\odot}$ (from the shock model), which is within a factor of 1.5 of that determined for SN2005af, also a massive Type II SN. This is a relatively small 
Table 6

Comparison between the Ejecta Masses of the SNR E0102 and Nucleosynthesis Yields

\begin{tabular}{|c|c|c|c|c|c|c|c|}
\hline & \multicolumn{3}{|c|}{$\operatorname{Ne}\left(M_{\odot}\right)$} & \multicolumn{3}{|c|}{$\mathrm{O}\left(M_{\odot}\right)$} & $\operatorname{Ar}\left(M_{\odot}\right)$ \\
\hline Infrared ejecta mass & \multicolumn{3}{|c|}{$\mathbf{0 . 0 4 2}$ (from shock model) } & \multicolumn{3}{|c|}{0.78} & $\ldots$ \\
\hline X-ray ejecta mass & \multicolumn{3}{|c|}{$0.1-2^{\mathrm{a}}\left(\mathbf{0 . 8 4 ^ { \mathrm { b } }}\right)$} & \multicolumn{3}{|c|}{$0.3-6^{\mathrm{a}}$} & $\ldots$ \\
\hline Total mass of infrared and X-ray ejecta & \multicolumn{3}{|c|}{$0.14-2.4\left(\mathbf{0 . 8 8 2}^{\mathrm{b}}\right)$} & \multicolumn{3}{|c|}{$1.08-6.78$} & $\ldots$ \\
\hline Nucleosynthesis models ${ }^{c}$ & A & B & $\mathrm{C}$ & A & $\mathrm{B}$ & $\mathrm{C}$ & A \\
\hline $13 M_{\odot}$ model & 0.028 & 0.023 & 0.064 & 0.21 & 0.151 & 0.308 & 0.0028 \\
\hline $15 M_{\odot}$ model & 0.039 & 0.033 & 0.049 & 0.433 & 0.368 & 0.520 & 0.0063 \\
\hline $20 M_{\odot}$ model & 0.257 & 0.191 & $\ldots$ & 1.480 & 0.800 & $\ldots$ & 0.004 \\
\hline $25 M_{\odot}$ model & 0.651 & 0.594 & 0.565 & 3.000 & 2.997 & 1.386 & 0.007 \\
\hline $35 M_{\odot}$ model & $\ldots$ & $\ldots$ & 1.366 & $\ldots$ & $\ldots$ & 3.891 & 0.040 \\
\hline $40 M_{\odot}$ model & $\ldots$ & 0.720 & $\ldots$ & $\ldots$ & 9.110 & $\ldots$ & $\ldots$ \\
\hline
\end{tabular}

Notes.

${ }^{\text {a }}$ From Flanagan et al. (2004).

b Estimates from our shock model. Note that our shock model matches the infrared lines.

c The model nucleosynthesis yields are taken from Thielemann et al. 1996 (A), Nomoto et al. 1997 (B), and Chieffi \& Limongi 2004 (C). The best estimate and matching nucleosynthesis yields are written in bold.

Table 7

Comparison of Elements Detected ${ }^{\mathrm{a}}$ in E0102 and Cas A

\begin{tabular}{|c|c|c|c|c|c|c|c|c|c|c|c|c|}
\hline Wavelength & SNR & $\mathrm{O}$ & $\mathrm{Ne}$ & $\mathrm{Mg}$ & $\mathrm{Si}$ & $\mathrm{S}$ & $\mathrm{Fe}$ & $\mathrm{Ar}$ & $\mathrm{Ca}$ & $\mathrm{H}$ & $\mathrm{He}$ & References $^{\mathrm{b}}$ \\
\hline \multirow[t]{2}{*}{ Infrared } & E0102 & $\mathrm{Y}$ & $\mathrm{Y}$ & $\ldots$ & $\mathrm{U}$ & $\mathrm{U}$ & $\mathrm{pN}$ & $\mathrm{N}$ & $\ldots$ & $\mathrm{N}$ & $\mathrm{N}$ & This paper \\
\hline & Cas A & $\mathrm{Y}$ & $\mathrm{Y}$ & $\ldots$ & $\ldots$ & $\ldots$ & $\mathrm{Y}$ & $\mathrm{Y}$ & $\ldots$ & $\ldots$ & $\ldots$ & 1,2 \\
\hline \multirow[t]{2}{*}{ Optical } & E0102 & $\mathrm{Y}$ & $\mathrm{Y}$ & $\mathrm{Y}$ & $\mathrm{pN}$ & $\mathrm{pN}$ & $\mathrm{pN}$ & $\mathrm{N}$ & $\mathrm{N}$ & $\mathrm{N}$ & $\mathrm{N}$ & 3 \\
\hline & Cas A & $\mathrm{Y}$ & $\mathrm{U}$ & $\mathrm{U}$ & $\mathrm{U}$ & $\mathrm{Y}$ & $\mathrm{N}$ & $\mathrm{Y}$ & $\mathrm{N}$ & $\mathrm{Y}^{*}$ & $\ldots$ & 4,5 \\
\hline \multirow[t]{2}{*}{ X-rays } & E0102 & $\mathrm{Y}$ & $\mathrm{Y}$ & $\mathrm{Y}$ & $\mathrm{Y}$ & $\mathrm{U}$ & $\mathrm{U}$ & $\mathrm{N}$ & $\mathrm{Y}$ & $\ldots$ & $\ldots$ & 6 \\
\hline & Cas A & $\mathrm{Y}$ & $\mathrm{Y}$ & $\mathrm{Y}$ & $\mathrm{Y}$ & $\mathrm{Y}$ & $\mathrm{Y}$ & $\mathrm{Y}$ & $\ldots$ & $\ldots$ & $\ldots$ & 7 \\
\hline
\end{tabular}

Notes.

a Y: detected, U: uncertain, pN: probably not detected, N: not detected, * indicates that the emission is likely from CSM instead of from ejecta (see the text for details).

b (1) Ennis et al. 2006, (2) Douvion et al. 2001, (3) Blair et al. 2000, (4) Fesen et al. 2006, (5) Fesen \& Gunderson 1996, (6) Flanagan et al. 2004, and (7) Hwang et al. 2000.

disparity given that there may be differences in the progenitor mass, the respective physical conditions of Ne-emitting gas, and thermodynamic history of $\mathrm{Ne}$ gas, and also does not account for hotter and more ionized $\mathrm{O}$ ejecta that are observed at shorter wavelengths.

We detected no infrared $\mathrm{H}$ lines from E0102, though a few do fall in the IRS spectral band. $\mathrm{H}$ lines are present in the optical spectra of E0102, but it is not likely that they are associated with the SN ejecta (Blair et al. 2000). For the purpose of estimating the $\mathrm{Ne}$ abundance relative to solar (or cosmic) values, however, we adopt the $\mathrm{H} \alpha$ line flux $5.94 \times 10^{-16} \mathrm{erg} \mathrm{s}^{-1} \mathrm{~cm}^{-2}$ and the upper limit of $\mathrm{H} \beta$ line flux of $1 \times 10^{-16} \mathrm{erg} \mathrm{s}^{-1} \mathrm{~cm}^{-2}$ reported by Blair et al. (2000) in order to obtain an upper limit on the $\mathrm{H}$ column density. At large optical depth, $4 \pi j(\mathrm{H} \beta) /\left(N_{p} N_{e}\right)$ is a constant for a given temperature that varies by only a factor of 5 over the relevant temperature range (Osterbrock 1989). Taking the path length to be the aperture beam size of $1^{\prime \prime}(0.299 \mathrm{pc})$, the $\mathrm{H}$ column $N$ is inferred to be $<7 \times 10^{13} \mathrm{~cm}^{-2}$. The Ne abundance in E0102 $([\mathrm{Ne} / \mathrm{H}])$ using the $\mathrm{Ne}$ column density of $4 \times 10^{16} \mathrm{~cm}^{-2}$ is then $>1.9 \times 10^{-3}$, while the solar value $\left([\mathrm{Ne} / \mathrm{H}]_{\odot}\right)$ is $1.2 \times 10^{-4}$, so $[\mathrm{Ne} / \mathrm{H}] /[\mathrm{Ne} / \mathrm{H}]_{\odot}>18$ if the optical $\mathrm{H} \alpha$ is associated with E0102. More likely, that is not the case, so this is a lower limit for the abundance of $\mathrm{Ne}$ relative to solar values.

We also calculated the column density of [O IV] using five levels for [O IV] lines and the atomic data of Tayal (2006). The column density inferred from the observed [O IV] line brightness is $1.5 \times 10^{13} \mathrm{~cm}^{-2}$. The equivalent oxygen mass is $8 \times 10^{-5} M_{\odot}$.
We also estimated the column densities and masses of [O I], [O II], [O III], and [O v] using the derived column density of [O IV], the Saha equation, and assuming that the oxygen gas has the same temperature as the Ne gas. The total mass of oxygen in the IRS aperture is thus inferred to be $0.26 M_{\odot}$. The calculated mass is quite sensitive to the second component temperature, however. For example, a temperature of $7000 \mathrm{~K}$ rather than $9100 \mathrm{~K}$ reduces the $\mathrm{O}$ mass a factor of 2 . The uncertainty in the estimated oxygen mass may thus be a factor of a few. To obtain the total oxygen mass for the entire remnant we scale the masses above by the same 3.30 multiplicative aperture correction factor used for $\mathrm{Ne}$. The total oxygen mass estimated for the entire remnant is then approximately $0.78 M_{\odot}$, as shown in Table 6. Note that oxygen mass estimate is much less accurate than that of $\mathrm{Ne}$ because we only have one observed oxygen line.

\subsection{Ejecta Mass and Nucleosynthesis Yields}

It is evident that the infrared census of the $\mathrm{Ne}$ and $\mathrm{O}$ ejecta mass is incomplete because the ejecta that emit at optical and $\mathrm{X}$-ray wavelengths are more ionized and at higher temperatures. infrared emission traces dense and relatively low-temperature ejecta as indicated by our line diagnostics, whereas X-rays trace much hotter and lower density coronal plasmas. The total neon and oxygen masses that we estimate from the infrared observations and shock models are compared with the nucleosynthesis yields of massive stars in Thielemann et al. 

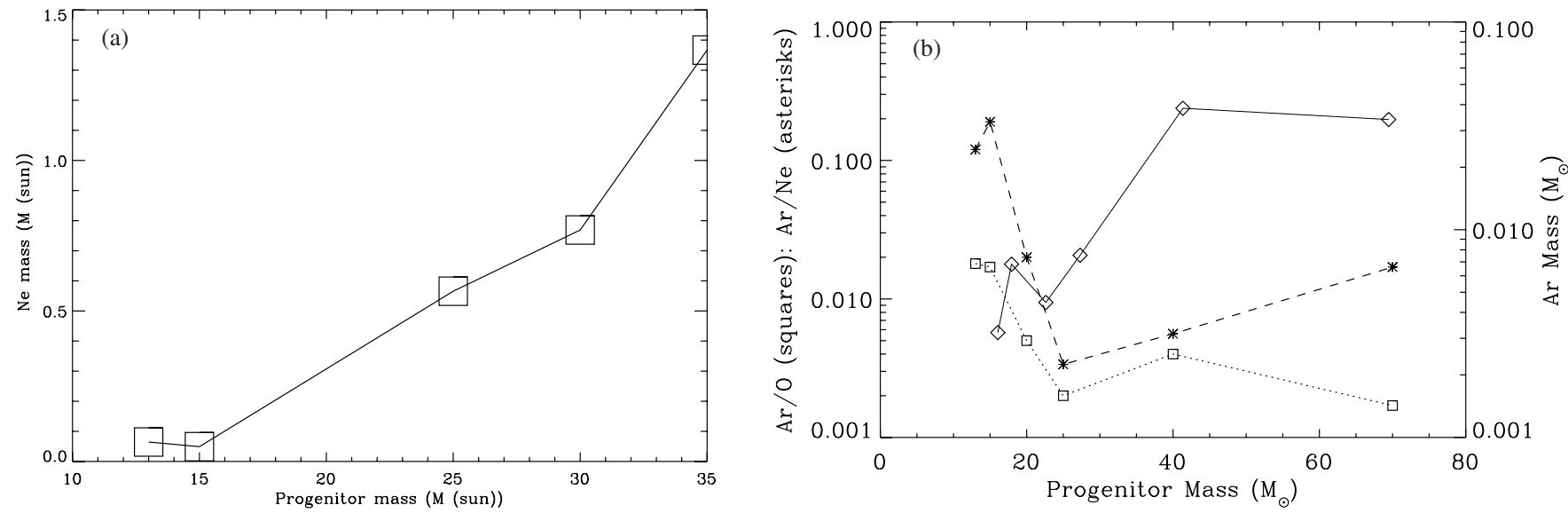

Figure 21. (a) Nucleosynthesis yields of Ne mass depending on the progenitor mass. (b) Nucleosynthesis yields of $\mathrm{Ar} / \mathrm{Ne}$ (dashed line), Ar/O (dotted line) ratios, and Ar mass (solid line) depending on the progenitor mass.

(1996), Nomoto et al. (1997), and Chieffi \& Limongi (2004) as summarized in Table 6.

The He- and $\mathrm{H}$-like ions that dominate the X-ray emission are inferred to have masses of $2 M_{\odot}$ for $\mathrm{Ne}$ and $6 M_{\odot}$ for $\mathrm{O}$ in the Chandra study of Flanagan et al. (2004); these indicate a progenitor mass of $32 M_{\odot}$. These mass estimates, however, are subject to uncertainties in the composition of the underlying continuum. Flanagan et al. (2004) make their mass estimate assuming that the ejecta do not include light elements, but if this is not the case, Flanagan et al. (2004) note that the X-ray-derived ejecta mass could be a factor of 20 lower, at 0.1 and $0.3 M_{\odot}$, for $\mathrm{Ne}$ and $\mathrm{O}$, respectively. By comparison, our estimates for the $\mathrm{Ne}$ and $\mathrm{O}$ ejecta masses from the infrared observations are $0.042 M_{\odot}$ and $0.78 M_{\odot}$, respectively. In the optical/UV analysis of Blair et al. (2000), relative line strengths and shock models favor a progenitor of $25 M_{\odot}$, but no attempt is made to estimate ejecta masses for the entire remnant.

If we take the lower set of values for the X-ray-emitting ejecta masses, we determine the total ejecta mass determined from the combined infrared and X-ray analysis to be $>0.142 M_{\odot}$ for $\mathrm{Ne}$ and $>1.08 M_{\odot}$ for $\mathrm{O}$. Taking the higher X-ray values, these masses are $2.04 M_{\odot}$ for $\mathrm{Ne}$ and $6.8 M_{\odot}$ for O. As a comparison, the X-ray-emitting Ne mass predicted by our shock model is $\sim 0.84 M_{\odot}$, though we note that this model was matched to the infrared Ne line emission only. Taken together, the inferred ejecta masses favor a progenitor mass of $30(25-40) M_{\odot}$ as shown in Figure 21(a) and Table 6, but are not strongly constraining. The infrared $\mathrm{Ne} / \mathrm{O}$ mass $(>0.13)$ ratio by itself favors a progenitor mass greater than $25 M_{\odot}$, as do the total masses of $\mathrm{Ne}$ and $\mathrm{O}$ when we take the lower range of the X-ray-emitting ejecta mass corresponding to the presence of light elements in the ejecta. This is not surprising given that the infrared ejecta mass makes up a significant fraction of the total mass (30\% for $\mathrm{Ne}$, and $70 \%$ for $\mathrm{O}$ ) if the X-ray ejecta masses are taken to be at this lower limit. Contrariwise, the infrared-emitting masses make up a very low fraction of the total ejecta mass ( $2 \%$ for $\mathrm{Ne}$ and $12 \%$ of $\mathrm{O}$ ) if we take the higher X-ray ejecta masses corresponding to pure heavy elements, and the inferred progenitor mass is then necessarily in agreement with that obtained in the X-ray analysis by Flanagan et al. (2004).

The set of line-emitting ejecta elements is very different for E0102 and Cas A (see Table 7), although both are young core-collapse SNRs with optical emission from highvelocity $\mathrm{O}$ ejecta. In $\mathrm{E} 0102$, the Ar lines are apparently absent in the infrared, and the Si and S lines are weak. Cas A, by contrast, shows very strong Ar emission as well as strong silicate $(21 \mu \mathrm{m}$ peak) dust emission (see the comparison in Table 7). In addition, Cas A shows unshocked, centrally located Si and S ejecta, whereas E0102 shows no indication of any unshocked ejecta. The differences in the $\mathrm{X}$-ray line emission from these two remnants are in line with those seen in the infrared. E0102 is dominated by $\mathrm{O}$ and $\mathrm{Ne}$ (Flanagan et al. 2004; Sasaki et al. 2006), while Cas A is dominated by $\mathrm{Si}, \mathrm{S}, \mathrm{Ar}, \mathrm{Ca}$, and $\mathrm{Fe}$. The ejecta temperatures in $\mathrm{Cas}$ $\mathrm{A}$ are higher, and presumably the $\mathrm{O}$ is too ionized to emit appreciable line emission, although Cas A is also subject to much higher interstellar absorption. And in spite of being considerably younger than E0102 (at an age of $330 \mathrm{yr}$ ), Cas A has a reverse shock that is already traversing the innermost ejecta layers. The key to this difference may be the extensive mass loss experienced by the Cas A progenitor, which probably had a binary companion (Young et al. 2006). Cas A is likely to have exploded at only $4 M_{\odot}$ after having started at $\sim 25 M_{\odot}$ on the main sequence (e.g., Laming \& Hwang 2003). This difference in the mass-loss history of the progenitors would also affect the SN nucleosynthesis and subsequent evolution of the remnant.

It is thus possible that the explosion of E0102 simply did not produce a significant mass of Ar. Certainly, variations of factors of 5-20 in Ar mass are evident in various $\mathrm{SNe}$ (for example, see the comparison of SN 2005af to SN 1987A and SN2004dj in Kotak et al. 2006). Nucleosynthesis models likewise predict a wide range of Ar masses, with more massive progenitors producing significantly more Ar. The yields range from 0.001 to $0.07 M_{\odot}$ for $25-40 M_{\odot}$ progenitors, with corresponding $\mathrm{Ar} / \mathrm{Ne}$ mass ratios of 0.001-0.1 (Figure 21). If we scale the predicted $\mathrm{Ar} / \mathrm{Ne}$ mass ratios to the total infrared $\mathrm{Ne}$ ejecta mass of $6.5 \times 10^{-3} M_{\odot}$, the models predict an infrared-emitting Ar mass between $6.5 \times 10^{-6}$ to $6.5 \times 10^{-4} M_{\odot}$.

We can use the detection limit of $3 \times 10^{-8} \mathrm{erg} \mathrm{s}^{-1} \mathrm{~cm}^{-2} \mathrm{sr}^{-1}$ for [Ar II] at $6.985 \mu \mathrm{m}$ to estimate the corresponding mass of $\mathrm{Ar}$ in E0102. Using two excitation levels of Ar, the column density of $\mathrm{Ar}$ is $1 \times 10^{10} \mathrm{~cm}^{-2}$ if we assume that $\mathrm{Ar}$ is at the same temperatures as $\mathrm{Ne}$. Since it is likely that the temperature and density could actually be quite different from those values, we consider a range of temperatures from 600 to $20,000 \mathrm{~K}$ and densities from $10^{3} \mathrm{~cm}^{-2}$ to $10^{5} \mathrm{~cm}^{-2}$. Including a factor of 3 correction for the IRS aperture, the final upper limit for the Ar mass ranges from $1.5 \times 10^{-8}$ to $1 \times 10^{-5} M_{\odot}$. The upper end of this mass range overlaps the range of the expected 
infrared-emitting Ar mass estimated above. It is thus plausible that the current nondetection of Ar is simply be due to limited sensitivity.

We cannot yet exclude the possibility that the infraredemitting Ar are truly absent, however. The physical conditions of the infrared-emitting Ar are highly uncertain. Possibly, the reverse shock has simply not reached the deep layers containing $\mathrm{Si}, \mathrm{S}, \mathrm{Ar}$, and $\mathrm{Ca}$ ejecta. It is also possible that the Ar might be detected in a more complete set of deep observations. While the IRS mapping data are too shallow to detect faint lines, the existing deep IRS staring-mode spectrum covered only one-third of the remnant. Given that core-collapse remnants often show rather asymmetric ejecta distributions, it cannot be ruled out that deep observations at other positions in E0102 might reveal Ar ejecta.

\section{6. [Ne v] Lines: Comparison with Other Astronomical Objects}

Among various astronomical objects, highly ionized [ $\mathrm{Ne} \mathrm{v}]$ is not commonly detected in the infrared because of its high ionization potential $(97.11 \mathrm{eV})$, whereas lines of both [Ne II] and [Ne III] are common and often bright. In galaxies, the detection of [Ne V] $24.3 \mu \mathrm{m}$ line of comparable brightness to [Ne II] is often taken as a signature of active galactic nuclei (AGNs; Armus et al. 2007) because [Ne v] cannot be readily produced by $\mathrm{O}$ stars. In the Galaxy, planetary nebulae emit [Ne v] lines which are produced by white dwarfs with high temperatures (Rubin 2004; Rubin et al. 1997; van Hoof et al. 2000). E0102 is the first SNR which is identified as an infrared [Ne v] emitter.

Here, we compare the physical conditions of ionic lineemitting material such as [Ne v] in the ejecta of E0102 with those of other astronomical objects. The [Ne v] 14.3/24.3 $\mu \mathrm{m}$ ratio of 1.76 is similar to many planetary nebulae (Rubin 2004), an example of which is NGC 7027. E0102 falls at the extreme high end of the range (0.2-1.8) observed in AGNs (Dudik et al. 2007; Sturm et al. 2002). Both our (see Figure 12) and Dudik et al.'s calculations use the same collisional strengths from Griffin \& Badnell (2000), and we are able to reproduce the [Ne v] $14.3 / 24.3 \mu \mathrm{m}$ ratio plot (Figure 1 of Dudik et al. 2007). Our measurements of $[\mathrm{Ne} \mathrm{v}]$ ratios are not affected by the extinction correction, because the intrinsic extinction correction is very low at less than $0.1 \%$ for E0102. In the SNR E0102, [ $\mathrm{Ne} \mathrm{v}$ ] lines come largely from collisionally ionized and radiatively cooling zones, whereas [Ne II] is dominantly from the photoionized zone. In AGNs, both [ $\mathrm{Ne} \mathrm{v}]$ and [ $\mathrm{Ne}$ II] lines are shown to be from photoionization. The ratio of $[\mathrm{Ne} \mathrm{V}] /[\mathrm{Ne}$ II $]$ is only 0.06 for E0102 and about 1 for AGN.

\subsection{Dust Mass and Implication of Supernova Dust in Early Universe}

Our dust masses for E0102 are 8-18 times greater than the $8 \times 10^{-4} M_{\odot}$ mass estimated by Stanimirovic et al. (2005) from MIPS imaging, but the latter assumes a temperature of $120 \mathrm{~K}$ and typical interstellar dust composition. The inferred dust mass will depend on the composition and temperature of the dust, which can only be accurately identified with spectral data such as the IRS data used here. The difference between our dust mass for E0102 and that of Stanimirovic et al. (2005, hereafter S06) thus arises from a number of factors. First, the IRS spectra indicate that there are three composition and temperature components. While two of the three components have temperatures comparable to the $120 \mathrm{~K}$ assumed by S06, the third component had a much lower temperature $(55-60 \mathrm{~K})$, and as shown in Table 4, dominates the mass. Moreover, because of the differences in dust composition assumed, there are differences in the absorption coefficients used and also in the inferred density; the latter are generally higher for the grains we used here, with $\mathrm{MgSiO}_{3}$, and $\mathrm{Al}_{2} \mathrm{O}_{3}$ having solid-state densities of 3.2 and $2.45 \mathrm{~g} \mathrm{~cm}^{-3}$, respectively, compared with more typical ISM grains, e.g., graphite with $\rho=2.25 \mathrm{~g} \mathrm{~cm}^{-3}$.

Our estimated dust mass is also higher than those generally inferred for SNe. Possibly, it is easier to detect dust in YSNRs than in extragalactic SNe simply because the dust in $\mathrm{SNe}$ is too cold to be detected in the infrared by Spitzer, whereas the freshly synthesized dust in YSNRs has been heated by the reverse shock to observable temperatures. Another limitation for estimating the dust mass of extragalactic $\mathrm{SNe}$ is that the spatial resolution currently available with infrared telescopes for observing $\mathrm{SNe}$ is still poor, and that makes it difficult to distinguish between fresh dust formed in the ejecta and ISM/CSM dust. In the case of E0102, we were able to resolve and separate the ejecta and the forward-shocked materials, and found no significant infrared emission from the ISM heated dust.

The estimated total dust mass of 0.007 to $0.015 M_{\odot}$ for E0102 is a factor of 3 lower than the mass (from 0.02 to $0.054 M_{\odot}$ ) of Cas A (Rho et al. 2008), though of the same order of magnitude. We discuss a few possibilities for the discrepancy between our estimated dust mass from E0102 and the dust mass per SN predicted by models (e.g., Todini \& Ferrara 2001; Nozawa et al. 2003) to account for the dust in high redshifted galaxies. First, there may be more mass in dust at lower temperatures. Our wavelength coverage is only up to $40 \mu \mathrm{m}$, and the MIPS $70 \mu \mathrm{m}$ emission has not been detected due to confusion with the nearby $\mathrm{H}$ II region of N76 and the limited spatial resolution of the $70 \mu \mathrm{m}$ image, as shown by Stanimirovic et al. (2005). We could not constrain a temperature of $41 \mathrm{~K}$ with our IRS data, as shown in Model $\mathrm{C}$ of Table 4. Even if colder dust $(\ll 55 \mathrm{~K})$ exists, our observations would not be sensitive to it. The colder dust components have been detected in Cas A and Kepler (Dunne et al. 2009; H. Gomez et al. 2009, in preparation) suggests that they could be present in other SNRs. Second, there are large uncertainties in dust destruction rates. The dust mass of Cas A may explain the lower limit on the dust masses in high redshift galaxies, when assuming conservative dust destruction rates (Rho et al. 2008; Morgan \& Edmunds 2003). When a more moderate destruction rate is taken account, a much higher dust mass per $\mathrm{SN}$ is needed to explain the dust in the early universe (Dwek et al. 2007; Galliano et al. 2008). The grain disruption which occurs primarily in SN-shocks is known to be too efficient to explain the amount of dust in the ISM (Jones et al. 1994). However, new infrared observations of SN 1987A suggest that the destruction rate of the silicate grains is a factor of 2 smaller than previous values (Dwek et al. 2008), and that microsized grains can survive the passage of a SN shock wave (Slavin et al. 2004). Third, an inaccurate estimation of progenitor masses from ejecta could cause the discrepancy, since the initial mass function (IMF) of massive stars is rather steep. As discussed in Section 4.5, progenitor mass depends on accurate estimates of ejecta masses over a broad range of wavelengths. Fourth, if E0102 contains Fe-bearing dust, the dust mass would be higher because the density of Fe dust $(\rho=7.95)$ is three or more times higher than other grains. Since Fe dust (due to the shape of $Q_{\text {abs }}$ ) is featureless like carbon and similar to that of $\mathrm{Al}_{2} \mathrm{O}_{3}$ at low temperatures $(<100 \mathrm{~K})$, it is difficult to distinguish between $\mathrm{Fe}, \mathrm{C}$, and $\mathrm{Al}_{2} \mathrm{O}_{3}$. While 
we assume no Fe dust because Fe gas lines are not detected, a small contribution of Fe could significantly increase the dust mass. Fifth, some grain types may be larger than assumed, which would increase the inferred mass. Sixth, smaller-sized dust in the ejecta is preferentially destroyed behind the reverse shock as demonstrated by Nozawa et al. (2007); in the case of E0102, this has been ongoing for $1000 \mathrm{yr}$. Deep observations with future telescopes, in particular, far-infrared/submillimeter observations and theoretical models of the time evolution of dust that is formed in the ejecta would be needed to settle this issue.

\section{SUMMARY}

Using Spitzer IRS and IRAC, we have carried out a study of the infrared emission from dust and ejecta in E0102. Our results may be summarized as follows.

1. We have detected infrared emission from E0102 in both continuum and lines. These include bright lines of [Ne II] $(12.8 \mu \mathrm{m}),[\mathrm{Ne}$ III] $(15.5 \mu \mathrm{m})$, [O IV] and/or [Fe II] $(26 \mu \mathrm{m})$ [O IV] and/or [Fe II] $(26 \mu \mathrm{m})$, weak lines of [Ne V] $(14.3 \mu \mathrm{m}),[\mathrm{NeV}](24.3 \mu \mathrm{m})$, and [Ne III] $(36 \mu \mathrm{m})$, and possibly [S II], [S III] $(33.5 \mu \mathrm{m})$, [Si II] $(34.89 \mu \mathrm{m})$. The ejecta in E0102 are dominated by $\mathrm{Ne}$ and $\mathrm{O}$ in the infrared, as in the X-ray and optical.

2. The [Ne II] lines are broadened by $20 \%-100 \%$ above the instrumental resolution, implying a true velocity dispersion of about $2000-4500 \mathrm{~km} \mathrm{~s}^{-1}$. This is comparable to velocities measured for the optically emitting ejecta, and indicates that the infrared [Ne II] emission is also from ejecta.

3. The density of the infrared-emitting $\mathrm{Ne}$ ejecta is high. The collisionally excited line diagnostics using [ $\mathrm{Ne} \mathrm{v}]$ and [Ne III] line ratios imply that [Ne III] lines come from a low $(\sim 600 \mathrm{~K})$ temperature and $[\mathrm{Ne} \mathrm{v}]$ lines from high-density region. A shock model with a best velocity parameter of $200 \mathrm{~km} \mathrm{~s}^{-1}$ indicates that the [ $\left.\mathrm{Ne} \mathrm{v}\right]$ lines mainly come from the cooling zone, which is hotter dense region, whereas $[\mathrm{Ne}$ II] and [Ne III] lines are mainly from the photoionization zone with a cold temperature of 400-1000 K.

4. We detect a broad dust feature at $18 \mu \mathrm{m}$, which we attribute to $\mathrm{MgSiO}_{3}$ and $\mathrm{Si}$; the remainder of the dust continuum can be fitted with either carbon or $\mathrm{Al}_{2} \mathrm{O}_{3}$ grains. The spatial correspondence of the dust continuum and the ejecta lines indicates that the dust is freshly formed in the ejecta.

5. The continuum show strong correlation with ejecta of E0102, showing that dust has formed in the ejecta. We estimate a dust mass of $7 \times 10^{-3}$ to $0.015 M_{\odot}$ that is higher than previous estimates by an order of magnitude, but smaller than that of Cas A.

6. We estimate the mass of infrared-emitting $\mathrm{Ne}$ and $\mathrm{O}$ ejecta. We estimate infrared-emitting Ne ejecta mass of $0.014 M_{\odot}$ and a total $\mathrm{Ne}$ mass of $1.26 M_{\odot}$ derived from the bestshock models. Together with the X-ray masses, these imply a progenitor mass of $\sim 30 M_{\odot}$.

For the future, detailed modeling to obtain accurate ejecta masses for young remnants such as Cas A and E0102 will be helpful to understand the production of elements such as Ar deep in the ejecta and their physical conditions in the remnant. More broadly, the goal is to understand the progenitors and the explosions that produced these remnants. Infrared observations with Spitzer and ISO have allowed us to identify dust features in SNRs and SNe including Cas A and E0102. However, the presence of cold dust, which can only be identified in the far-infrared and submillimeter, is still uncertain due to limited angular resolution at these wavelengths. In the near-future, we expect Herschel observations will significantly advance our knowledge of cold dust. Sensitive observations will be very helpful to find and study the ejecta, so deep infrared mid- and near-infrared observations with Spitzer and with future missions such as James Webb Space Telescope will significantly advance our understanding of the questions of nucleosynthesis and dust formation in $\mathrm{SN}$ explosions.

We thank Haley Gomez for insightful, helpful comments on the manuscript. J.R. thanks Pierre-Olivier Lagage, Sacha Hony, and Anne Decourchelle for fruitful discussion on dust and SN ejecta during her visit to Saclay. We thank Lee Armus and David Shupe for sharing the results of the IRS wavelength calibration on the IRS instrumental line widths, and Steven Finkelstein and William Blair for sharing their digitalized formats of HST and the optical images, and Shaun Amy for a radio image. This work is based on observations made with the Spitzer Space Telescope, which is operated by the Jet Propulsion Laboratory, California Institute of Technology, under NASA contract 1407. Partial support for this work was provided by NASA through an GO award issued by JPL/Caltech.

\section{REFERENCES}

Amy, S. W., \& Ball, L. 1993, ApJ, 411, 761

Arendt, R. G., Dwek, E., \& Moseley, S. H. 1999, ApJ, 521, 234 (ADM)

Armus, L., et al. 2007, ApJ, 656, 148

Beelen, A., et al. 2006, ApJ, 642, 694

Bertoldi, F., Carilli, C. L., Cox, P., Fan, X., Strauss, M. A., Beelen, A., Omont, A., \& Zylka, R. 2003, A\&A, 406, L55

Bianchi, S. 2009, in ASP Conf. Ser., Cosmic Dust-Near \& Far, ed. Th. Henning, E. Grun, \& J. Steinacker (San Francisco, CA: ASP), in press

Bianchi, S., \& Schneider, R. 2007, MNRAS, 378, 983

Blair, W. P., Raymond, J. C., Danziger, J., \& Matteucci, F. 1989, ApJ, 333, 812

Blair, W. P., et al. 2000, ApJ, 537, 667

Chevalier, R. A., \& Kirshner, R. P. 1978, ApJ, 219, 931

Chevalier, R. A., \& Kirshner, R. P. 1979, ApJ, 233, 154

Chieffi, A., \& Limongi, M. 2004, ApJ, 608, 405

Clayton, D. D. 1982, in Lunar Planetary Science Conf., (A82-31677 15-91) (New York: Pergamon), 1781

Clayton, D. D., Arnett, K. J., \& Meyer, B. S. 1997, ApJ, 486, 824

Clayton, D. D., \& Nittler, L. R. 2004, ARA\&A, 42, 39

Deneault, E. A.-N., Clayton, D. D., \& Heger, A. 2003, ApJ, 594, 312

Deneault, E. A.-N., Clayton, D. D., \& Meyer, B. S. 2006, ApJ, 638, 234

Douvion, T., Lagage, P. O., \& Pantin, E. 2001, A\&A, 369, 589

Draine, B. T., \& Lee, H. M. 1984, ApJ, 285, 89

Dudik, R. R, Weingartner, J. C., Satyapal, S., Sisher, J., Dudley, C. C., \& O'Halloran, B. 2007, ApJ, 664, 71

Dunne, L., Eales, S., Ivison, R., Morgan, H., \& Edmunds, M. 2003, Nature, 424, 285

Dunne, L., et al. 2009, MNRAS, 394, 1307

Dwek, E., Galliano, F., \& Jones, A. 2007, Nuovo Cimento B, 122, 959

Dwek, E., et al. 2008, ApJ, 676, 1029

Ellison, D. C., Drury, L. O., \& Meyer, J. 1997, ApJ, 487, 197

Elmhamdi, A., et al. 2003, MNRAS, 338, 939

Ennis, J., et al. 2006, ApJ, 652, 376

Ferland, G. J., Korista, K. T., Verner, D. A., Ferguson, J. W., Kingdon, J. B., \& Verner, E. M. 1998, PASP, 110, 761

Fesen, R., \& Gunderson, K. S. 1996, ApJ, 470, 967

Fesen, R. A., et al. 2006, ApJ, 645, 283

Finkelstein, S. L., et al. 2006, ApJ, 641, 919

Flanagan, K. A., Canizares, C. R., Dewey, D., Houck, J. C., Fredericks, A. C., Schattenburg, M. L., Markert, T. H., \& Davis, D. S. 2004, ApJ, 605, 230 Gaetz, T. J., Butt, Y. M., Edgar, R. J., Eriksen, K. A., Plucinsky, P. P., Schlegel, E. M., \& Smith, R. K. 2000, ApJ, 534, L47

Galliano, F., Dwek, E., \& Chanial, P. 2008, ApJ, 672, 214

Griffin, D. C., \& Badnell, N. R. 2000, At. Mol. Opt. Phys., 33, 4389

Hilditch, R. W., Howarth, I. D., \& Harries, T. J. 2005, MNRAS, 357, 304 Hofmeister, et al. 2003, MNRAS, 345, 16 
Hughes, J. P., \& Rakowski, C. E., Decourchelle 2000, ApJ, 543, L61

Hwang, U., Holt, S. S., \& Petre, R. 2000, ApJ, 537, L119

Jones, A. P., Tielens, A. G. M., Hollenbach, D. J., \& McKee, C. F. 1994, ApJ, 433, 797

Keller, S. C., \& Wood, P. R. 2006, ApJ, 642, 834

Kotak, R., et al. 2006, ApJ, 651, L117

Kozasa, T., Hasegawa, H., \& Nomoto, K. 1989, ApJ, 344, 325

Kozasa, T., Hasegwaa, H., \& Nomoto, K. 1991, A\&A, 249, 474

Kozasa, T., Nozawa, T., Tominaga, N., Hideyuki, U., Maeda, H., \& Nomoto, K. 2009, in ASP Conf. Ser., Cosmic Dust-Near and Far, ed. Th. Henning, E. Grun, \& J. Steinacker (San Francisco, CA: ASP), in press (arXiv:0903.0217v2)

Krause, O., et al. 2004, Nature, 432, 596

Lagage, P. O., Claret, A., Ballet, J., Boulanger, F., Cesarsky, C. J., Cesarsky, D., Fransson, C., \& Pollock, A. 1996, A\&A, 315, L273

Laming, J. M., \& Hwang, U. 2003, ApJ, 597, L347

Lucy, L. B., Danziger, I. J., Gouiffes, C., \& Bouchet, P. 1989, in IAU Coll. 120, Structure and Dynamics of Interstellar Medium, ed. G. Tenorio-Tagle et al. (New York: Springer), 164

Lucy, L. B., Danziger, I. J., Gouiffes, C., \& Bouchet, P. 1991, in Supernovae, ed. S. E. Woosley (New York: Springer), 82

Messenger, S., Sandford, S., \& Brownlee, D. 2006, in Meteorites and the Early Solar System II, ed. D. S. Lauretta \& H. Y. McSween, Jr. (Tucson, AZ: Univ. Arizona Press), 187

Morgan, H. L., Dunne, L., Eales, S. A., Ivison, R. J., \& Edmunds, M. G. 2003, ApJ, 597, L33

Morgan, H. L., \& Edmunds, M. G. 2003, MNRAS, 343, 427

Nomoto, K., Hashimoto, M., Tsujimoto, T., Thielemann, F.-K., Kishimoto, N., Kubo, Y1., \& Nakasato, N. 1997, Nucl. Phys., 616, 79

Nozawa, T., Kozasa, T., Habe, A., Dwek, E., Umeda, H., Tominaga, N., Maeda, K., \& Nomoto, K. 2007, ApJ, 666, 955
Nozawa, T., Kozasa, T., Umeda, H., Maeda, K., \& Nomoto, K. 2003, ApJ, 598, 785 (N03)

Nozawa, T., et al. 2008, ApJ, 684, 1343

Osterbrock, D. E. 1989, Astrophysics of Gaseous Nebulae and Active Galactic Nuclei (Sausalito, CA: Univ. Science Books)

Raymond, J. C. 1979, ApJS, 39, 1

Raymond, J. C., \& Smith, B. W. 1977, ApJS, 35, 419

Rho, J., Jarrett, T. H., Reach, W. T., Gomez, H., \& Andersen, M. 2009, ApJ, 693, L39

Rho, J., Kozasa, T., Reach, W. T., Smith, J. D., Rudnick, L., DeLaney, T., Ennis, J. A., \& Gomez, H. 2008, ApJ, 673, 271

Rubin, R. H. 2004, in IAU Symp. 217, Recycling Intergalactic and Interstellar Matter, ed. P. A. Duc, J. Briane, \& E. Brinks (San Francisco, CA: ASP), 190

Rubin, R. H., Colgan, S. W. J., Hass, M. R., Lord, S. D., \& Simpson, J. P. 1997, ApJ, 479, 332

Sasaki, M., Gaetz, T. J., Blair, W. P, Edgar, R., Morse, J. A, Plucinsky, P. P., \& Smith, R. K. 2006, ApJ, 642, 260

Slavin, J. D., Jones, A. P., \& Tielens, A. G. G. M. 2004, ApJ, 614, 796

Stanimirovic, S., et al. 2005, ApJ, 632, 103

Sturm, E., Lutz, D., Verma, A., Netzer, H., Sternberg, A., Moorwood, A. F. M., Oliva, E., \& Genzel, R. 2002, A\&A, 393, 821

Tappe, A., Rho, J., \& Reach, W. T. 2006, ApJ, 653, 267

Tayal, S. S. 2006, ApJS, 166, 634

Thielemann, F.-K., Nomoto, K., \& Hashimoto, M.-A. 1996, ApJ, 460, 408

Todini, P., \& Ferrara, A. 2001, MNRAS, 325, 726 (TF)

Tuohy, I. R., \& Dopita, M. 1983, ApJ, 268, L11

van Hoof, P. A. M., Beinterma, D. A., \& Ferland, G. J. 2000, A\&A, 354, L41

Wang, R., et al. 2008, ApJ, 687, 848

Wilson, T. L., \& Batrla, W. 2005, A\&A, 430, 561

Woosley, S. E., \& Weaver, T. A. 1995, ApJS, 101, 181

Young, P. A., et al. 2006, ApJ, 640, 891 